\title{
Macrolide Allergic Reactions
}

\author{
Kristy M. Shaeer ${ }^{1, *,+}$, Elias B. Chahine ${ }^{2,+}$, Sheeba Varghese Gupta ${ }^{3,+}$ and Jonathan C. Cho ${ }^{4,+}$ \\ 1 Department of Pharmacotherapeutics and Clinical Research, University of South Florida College of \\ Pharmacy, Tampa, FL 33612, USA \\ 2 Department of Pharmacy Practice, Lloyd L. Gregory School of Pharmacy, Palm Beach Atlantic University, \\ West Palm Beach, FL 33416, USA; elias_chahine@pba.edu \\ 3 Department of Pharmaceutical Sciences, University of South Florida College of Pharmacy, Tampa, FL 33612, \\ USA; svarghes@health.usf.edu \\ 4 Department of Clinical Sciences, Ben and Maytee Fisch College of Pharmacy, University of Texas at Tyler, \\ Tyler, TX 75799, USA; jcho@uttyler.edu \\ * Correspondence: kshaeer@health.usf.edu; Tel.: +1-813-974-0967 \\ $\dagger$ The authors contributed equally to the present work.
}

Received: 2 August 2019; Accepted: 11 September 2019; Published: 18 September 2019 updates

\begin{abstract}
Macrolides are antimicrobial agents that can be used to treat a variety of infections. Allergic reactions to macrolides occur infrequently but can include minor to severe cutaneous reactions as well as systemic life-threatening reactions such as anaphylaxis. Most reports of allergic reactions occurred in patients without prior exposure to a macrolide. Cross-reactivity among macrolides may occur due to the similarities in their chemical structures; however, some published literature indicates that some patients can tolerate a different macrolide. Most published reports detailed an allergic reaction to erythromycin. Desensitization protocols to clarithromycin and azithromycin have been described in the literature. The purpose of this article is to summarize macrolide-associated allergic reactions reported in published literature. An extensive literature search was conducted to identify publications linking macrolides to hypersensitivity reactions.
\end{abstract}

Keywords: macrolides; allergy; azithromycin; erythromycin; clarithromycin; fidaxomicin; desensitization

\section{Introduction}

One of the most common causes of medication allergies among adults and children is antibiotics [1]. These allergic reactions can range from immediate to non-immediate (delayed) hypersensitivity reactions. Immediate reactions are typically IgE-mediated and can cause clinical manifestations that include urticaria, angioedema, and anaphylaxis [1,2]. Non-immediate reactions are frequently T-cell mediated and can lead to various degrees of cutaneous symptoms in patients [1,2]. Although many of the reported antibiotic allergies are from the beta-lactam class, cases of allergic reactions to macrolide antibiotics have been documented [1-4]. However, hypersensitivity reactions resulting from macrolide use occurs infrequently (0.4-3\%) [4]. Since macrolide allergies are uncommon, there is a lack of recent data reviewing macrolide allergies and management of those allergies. This paper will review the medicinal chemistry, indications, reported allergic reactions, and desensitization protocols associated with macrolide antimicrobials.

\section{Medicinal Chemistry}

\subsection{Macrolide Structure}

The structure of macrolides consists of a large lactone ring that varies in size from 12 to 18 atoms. Sugar molecules are attached to the lactone ring with glycosidic bonds. Macrolide antibiotics are 
classified based on the number of atoms in the lactone ring; 14-membered lactones (erythromycin and clarithromycin), 15-membered lactones (azithromycin), ketolide (telithromycin) and 18-membered lactone (fidaxomicin). All clinically available macrolides, with the exception of erythromycin and fidaxomicin, are either synthetically or semi-synthetically generated. Natural macrolides have instability in the gastric environment, resulting in undesired pharmacokinetic properties, such as incomplete absorption resulting in decreased bioavailability [5].

\subsection{Mechanism of Action}

All macrolides except for fidaxomicin block bacterial protein synthesis by binding reversibly to the $23 \mathrm{~S}$ ribosomal RNA (rRNA) in the 50S-subunit of prokaryotic ribosomes [6]. Fidaxomicin exerts its bactericidal effects by inhibiting bacterial RNA polymerase at transcription initiation by binding to the DNA template-RNA polymerase complex [7].

\subsection{Structural Aspects behind Cross-Reactivity}

Allergic reactions to macrolides are relatively less common compared to other classes of antibiotics (Figure 1) [4]. Macrolides with a 14- membered lactone ring such as erythromycin and clarithromycin have been reported to express cross-reactivity in single case reports. The exact mechanism of hypersensitivity due to macrolides is not clearly understood [8]. Azithromycin is a semisynthetic derivative of erythromycin with a 15-membered lactone ring in its structure. Owing to azithromycin's structural similarity to erythromycin, cross-reactivity with erythromycin has also been reported. There is a lack of scientific evidence to support cross sensitization between various macrolide derivatives [8].

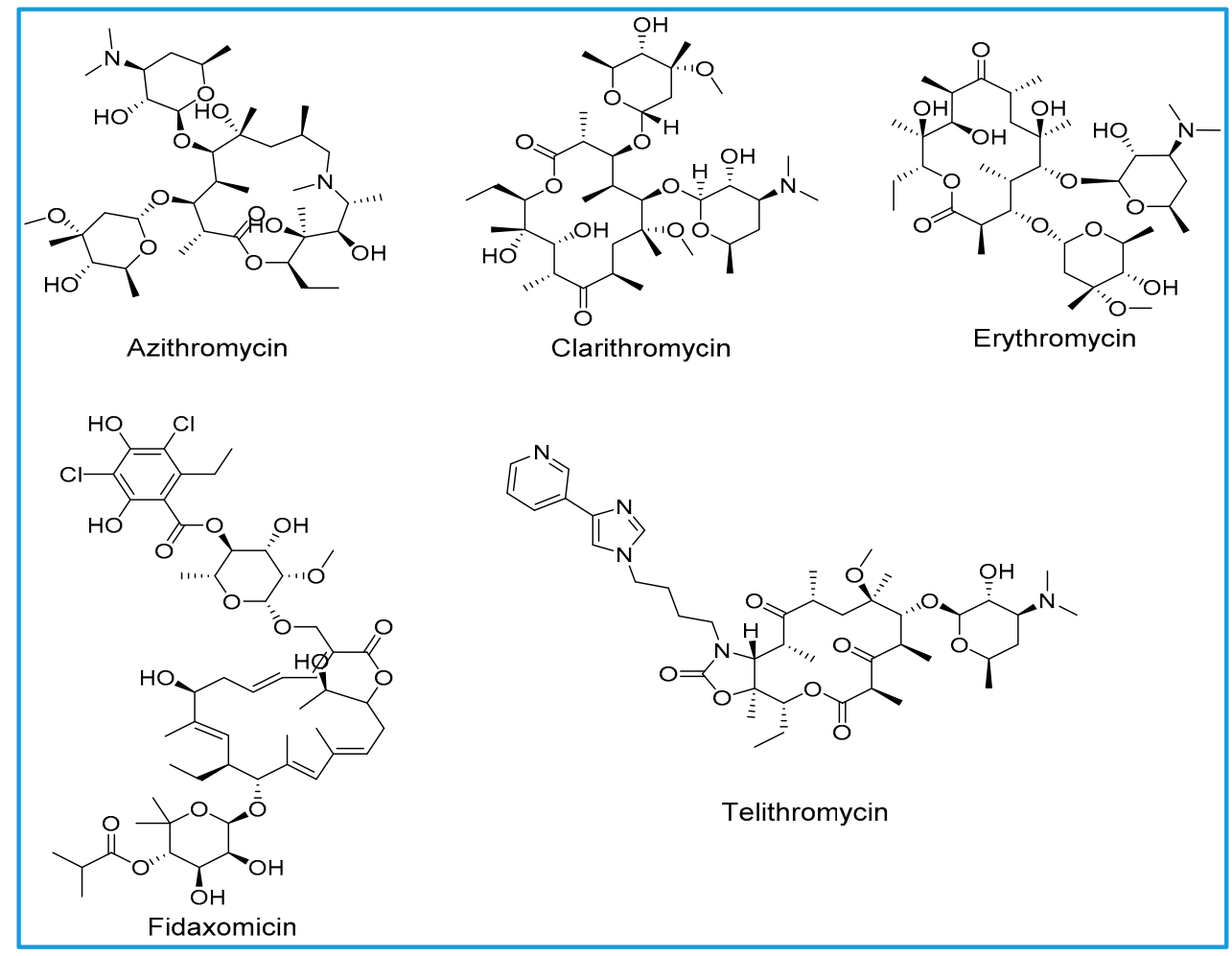

Figure 1. Chemical structures of macrolides.

\section{Place in Therapy}

Macrolides are a well-established class of antibiotics. They exhibit bacteriostatic activity against a wide range of gram-positive, gram-negative, and atypical bacteria [9]. Erythromycin, the macrolide with the longest use in practice, has a few remaining indications as the drug of choice given the 
rise in antibiotic resistance and the availability of more effective and safer antibiotics. Azithromycin and clarithromycin have largely replaced erythromycin in clinical practice because of their broader spectrum of activity, better pharmacokinetics profile, and fewer gastrointestinal adverse effects [9]. In addition, azithromycin is associated with fewer drug-drug interactions than erythromycin and clarithromycin [9].

Macrolides are the drugs of choice for the treatment of various atypical bacteria [9]. Erythromycin is the drug of choice for the treatment of diphtheria caused by Corynebacterium diphtheriae, although antitoxin is the primary treatment. Erythromycin is also the drug of choice for the treatment of infants with pneumonia caused by Chlamydia trachomatis. Azithromycin is the drug of choice for the treatment of trachoma, urethritis, and cervicitis caused by Chlamydia trachomatis and chancroid caused by Haemophilus ducreyi. Azithromycin with or without rifampin is also one of the regimens of choice for the treatment of Legionella pneumophila, which can cause serious atypical pneumonia. Clarithromycin is the drug of choice for the treatment of Helicobacter pylori as part of a combination regimen with amoxicillin and omeprazole. Clarithromycin in addition to amikacin is the regimen of choice for the treatment of infections caused by Mycobacterium fortuitum and Mycobacterium chelonae. Erythromycin and azithromycin are the drugs of choice for the treatment of infections caused by Bartonella henselae (cat scratch fever), Bartonella quintana (trench fever), Campylobacter jejuni (diarrhea), Chlamydia trachomatis (conjunctivitis and urethritis), and Ureaplasma urealyticum (urethritis). Azithromycin and clarithromycin are the drugs of choice for the treatment of infections caused by Mycobacterium avium complex as part of a combination regimen with ethambutol and rifabutin or monotherapy for primary or secondary prophylaxis. Lastly, all three macrolides are the drugs of choice for the treatment of pertussis (whooping cough) caused by Bordetella pertussis and atypical pneumonia caused by Chlamydophila pneumonia and Mycoplasma pneumoniae.

Many clinical practice guidelines recommend the use of macrolides for the empiric treatment of respiratory tract infections although antibiotic resistance among Streptococcus pneumoniae, Haemophilus influenzae, and Moraxella catarrhalis is on the rise [10-13]. Current guidelines recommend the empiric use of macrolides as the drugs of choice for the treatment of atypical pneumonia in children and community-acquired pneumonia in adults as monotherapy in the outpatient setting and as combination therapy with a beta-lactam in the inpatient setting $[10,11]$. Current guidelines recommend the empiric use of macrolides as alternative choices for the treatment of acute otitis media and streptococcal pharyngitis $[12,13]$.

Macrolides can also be used as alternative options for the treatment of various infections in patients who are not able to take the drugs of choice because of allergic reaction or intolerance [9]. For example, erythromycin can be used as an alternative to cephamycins as part of a combination regimen to prevent infections associated with colorectal surgeries or as an alternative to penicillins for the prevention of rheumatic fever. In rare circumstances, erythromycin can be used as an alternative to ciprofloxacin, doxycycline, and penicillins for the treatment of anthrax caused by Bacillus anthracis or as an alternative to tetracyclines for the treatment of infections caused by Lymphogranuloma venereum. More commonly, erythromycin can be used as an alternative to tetracyclines for the treatment of acne vulgaris. Azithromycin can be used as an alternative to ceftriaxone or fluoroquinolones for the treatment of typhoid fever caused by Salmonella typhi or as an alternative to fluoroquinolones for the treatment of diarrhea caused by Shigella dysenteriae. Azithromycin can also be used as an alternative to doxycycline and penicllins for the treatment of Lyme disease caused by Borrelia burgdorferi or as an alternative to clindamycin and quinine for the treatment of babesiois caused by Babesia microti. Azithromycin and clarithromycin can be used as an alternative to trimethoprim/sulfamethoxazole for the treatment of respiratory infections caused by Haemophilus influenzae. Lastly, all three macrolides can be used as an alternative to penicillins for the treatment of respiratory and skin and soft tissue infections caused by groups A, C, and G Streptococcus, Streptococcus pneumoniae, and Moraxella catarrhalis.

Fidaxomicin is a unique antibiotic, and represents the latest addition to the macrolides [14]. It exhibits bactericidal activity against Clostridoides difficile. Fidaxomicin is not systemically absorbed, is 
well tolerated, and is not associated with any known drug interactions. Clinical trials have shown that fidaxomicin is non-inferior to vancomycin for the treatment of Clostridioides difficile infection (CDI) and is associated with lower recurrence rates. Current CDI guidelines recommend the use of fidaxomicin for the treatment of initial severe, non-severe, and recurrent episodes [15].

\section{Published Allergic Reactions}

To gather relevant information, a literature search was performed using the PubMed, EBSCOhost, and Google Scholar electronic databases for articles published up to 17 May 2019, with restrictions for English language and human subjects. Search terms used to identify the included articles were macrolides, azithromycin, clarithromycin, erythromycin, fidaxomicin, hypersensitivity, allergy, rash, toxic epidermal necrosis, Stevens Johnson Syndrome, fixed drug eruption, maculopapular rash, exanthema, and desensitization. Articles about macrolides used as immunosuppressants (e.g., tacrolimus, everolimus, pimecrolimus, and sirolimus) and uncommonly or commercially unavailable were excluded (e.g., kitasamycin, josamycine midecamycin, roxithromycin, spiramycin, telithromycin, and troleandomycin). References of publications for which the full text was retrieved were also reviewed for additional literature sources.

In general, allergic reactions to macrolides reported in the literature are rare. Macrolides are available in a variety of dosage forms, and of those, topical, oral, intravenous, and ophthalmic formulations have been reported to cause an allergic reaction. The initial search for articles regarding macrolide hypersensitivity yielded 1895 citations. Following completion of all search strategies and terms a total of 120 reports were included and summarized in this review. The types of reactions for erythromycin, clarithromycin, azithromycin, and fidaxomicin are summarized in Tables 1-4. The included reports were published between 1958 and 2018, with reports from 27 different countries. Reported reactions occurred in a variety of patient populations, such as pediatrics $(n=50)$, adults $(n=105)$, and unknown $(n=20)$. Many providers tested patients to confirm the hypersensitivity $(n=79)$. Several of the reactions involved patients who had received a prior macrolide $(n=43)$ and of those 23 patients with repeated reactions. Repeated occupational exposures led to 10 subjects with cutaneous adverse reactions to azithromycin [16-18].

The breakdown of studies included 88 case reports, 12 case series, 2 cross-sectional, 1 double-blind, placebo-controlled, 5 prospective, and 12 retrospective. There were 58 publications describing erythromycin-associated allergic reactions [19-77]. There were 33 publications describing clarithromycin as a culprit for allergic reactions $[72,73,75,78-106]$. There were 31 published reports describing azithromycin associated allergic reactions [16-18,95,104,107-131]. Uniquely, the ophthalmic formulation of azithromycin was associated with contact dermatitis [119,123]. There was a single publication dedicated to a case series describing 12 patients who had hypersensitivity reactions to fidaxomicin [132]. Maculopapular exanthema eruptions developed in four subjects suffering from mononucleosis who were also on macrolide therapy $[43,109,111,122]$. Five cases involved the development of contact dermatitis with topical erythromycin $[26,35,51,56,58]$. Four cases resulted in a fatality secondary to the severe allergic reaction $[19,33,88,91]$. Fourteen patients were reported to have anaphylactic reactions to a macrolide $[19,23,59,66,73,74,81,98,100,108,121,132]$. Drug reaction with eosinophilia and systemic symptoms syndrome was reported in four patients who received azithromycin and one patient who received clarithromycin [105,118,120,124,129]. Erythromycin $(n=1)$, clarithromycin $(n=2)$, and azithromycin $(n=1)$ have been implicated in leukocytoclastic vasculitis $[60,79,84,113]$. Fourteen publications implicated a macrolide as the cause of an allergic reaction with subjects who received concomitant antimicrobials $[25,41,44-46,58,76,78,90,101,102,105,119,131]$. The most common concomitant agents were an aminoglycoside $(n=3)$, a beta-lactam $(n=3)$, and a sulfa $(\mathrm{n}=4)[41,44-46,78,90,101,102,119,131]$. It is possible that one of the concomitant antimicrobials could have elicited the allergic reaction. Two publications excluded a concomitant antimicrobial as the cause with allergy testing $[45,58]$. 
Table 1. Summary of published literature reporting erythromycin hypersensitivity.

\begin{tabular}{|c|c|c|c|c|c|c|c|}
\hline Reaction & Time of Onset $^{\#}$ & Demographics & Dosage Form & Concomitant Agents & Allergy Evaluation/Confirmation & Prior Sensitization & Notes \\
\hline Anaphylaxis [19] & $5 \min$ & $2 \mathrm{y} / \mathrm{oF}$ & IM & $\mathrm{N}$ & $\mathrm{N}$ & $\mathrm{N}$ & Fatal \\
\hline Urticaria [20] & 1 day & $24 \mathrm{y} / \mathrm{o} \mathrm{M}$ & IM & $\mathrm{N}$ & $\begin{array}{c}\mathrm{Y} \\
(\mathrm{IDT}+)\end{array}$ & $\mathrm{N}$ & \\
\hline MPR [21] & 5 days & $\mathrm{NR}(<18 \mathrm{y} / \mathrm{o})$ & $\mathrm{PO}$ & $\mathrm{N}$ & $\begin{array}{c}\mathrm{Y} \\
(\mathrm{DPT}+)\end{array}$ & $\mathrm{N}$ & \\
\hline MPR [21] & 2 days & $\mathrm{NR}(<18 \mathrm{y} / \mathrm{o})$ & $\mathrm{PO}$ & $\mathrm{N}$ & $\mathrm{N}$ & $\mathrm{N}$ & \\
\hline MPR [21] & NR & $\mathrm{NR}(<18 \mathrm{y} / \mathrm{o})$ & $\mathrm{PO}$ & $\mathrm{N}$ & $\begin{array}{c}\mathrm{Y} \\
\text { (DPT-) }\end{array}$ & $\mathrm{N}$ & \\
\hline MPR [22] & NR & NR & $\mathrm{PO}$ & $\mathrm{N}$ & $\mathrm{N}$ & $\mathrm{N}$ & \\
\hline $\begin{array}{l}\text { Anaphylaxis (dyspnea, } \\
\text { laryngeal edema) [23] }\end{array}$ & Several h & $60 \mathrm{y} / \mathrm{o} \mathrm{M}$ & $\mathrm{PO}$ & $\mathrm{N}$ & $\mathrm{Y}(\mathrm{MDT}+)$ & $\mathrm{N}$ & \\
\hline HSP [24] & $1-1.5$ days & $51 \mathrm{y} / \mathrm{o} \mathrm{M}$ & $\mathrm{PO}$ & $\mathrm{N}$ & $\mathrm{N}$ & $\mathrm{N}$ & \\
\hline FDE [25] & $12+h^{*}$ & $22 \mathrm{y} / \mathrm{o} \mathrm{M}$ & $\mathrm{PO}$ & $\begin{array}{c}\mathrm{Y} \\
\text { (terramycin) }\end{array}$ & $\mathrm{N}$ & $\begin{array}{c}\mathrm{Y} \\
\text { (E, with same reaction) }\end{array}$ & \\
\hline Urticaria [26] & 7 days & $7 \mathrm{y} / \mathrm{oF}$ & $\mathrm{PO}$ & $\mathrm{N}$ & $\begin{array}{c}\mathrm{Y} \\
(\mathrm{PT}-, \mathrm{ST}+)\end{array}$ & $\mathrm{N}$ & \\
\hline CD [26] & 21 days & $52 \mathrm{y} / \mathrm{o} \mathrm{F}$ & TOP & $\mathrm{N}$ & $\begin{array}{c}\mathrm{Y} \\
(\mathrm{PT}+)\end{array}$ & $\mathrm{N}$ & \\
\hline Cholestatic hepatitis [27] & 1.5 days & $53 \mathrm{y} / \mathrm{oF}$ & $\mathrm{PO}$ & $\mathrm{N}$ & $\begin{array}{c}\mathrm{Y} \\
(\mathrm{LTT}+)\end{array}$ & $\begin{array}{c}\mathrm{Y} \\
(\mathrm{E}, 2.5 \mathrm{yrs} \text { prior no reaction) }\end{array}$ & \\
\hline $\begin{array}{c}\text { MPR [28] } \\
\text { Hepatotoxicity }\end{array}$ & 1 day & $7 \mathrm{y} / \mathrm{o} \mathrm{M}$ & $\mathrm{PO}$ & $\mathrm{N}$ & $\mathrm{N}$ & $\begin{array}{c}\mathrm{Y} \\
\text { (28 days prior no reaction) }\end{array}$ & \\
\hline Rash [29] & 8 days & $51 \mathrm{y} / \mathrm{o} \mathrm{M}$ & IV & $\mathrm{N}$ & $\mathrm{N}$ & $\mathrm{N}$ & \\
\hline Rash [30] & 7 days & $18 \mathrm{y} / \mathrm{o} \mathrm{F}$ & $\mathrm{PO}$ & $\mathrm{N}$ & $\begin{array}{c}\mathrm{Y} \\
(\mathrm{DPT}+)\end{array}$ & $\mathrm{N}$ & \\
\hline Cholestatic hepatitis [31] & 17 days & $15 \mathrm{y} / \mathrm{o} \mathrm{F}$ & $\mathrm{PO}$ & $\begin{array}{c}\mathrm{Y} \\
\text { (propoxyphene) }\end{array}$ & $\mathrm{N}$ & $\mathrm{N}$ & \\
\hline Cholestatic hepatitis [31] & 7 days & $23 \mathrm{y} / \mathrm{o} \mathrm{M}$ & $\mathrm{PO}$ & $\mathrm{N}$ & $\mathrm{N}$ & $\mathrm{N}$ & \\
\hline Cholestatic hepatitis [31] & 1 day & $44 \mathrm{y} / \mathrm{o} \mathrm{F}$ & $\mathrm{PO}$ & $\begin{array}{c}\mathrm{Y} \\
\text { (propoxyphene) }\end{array}$ & $\mathrm{N}$ & $\begin{array}{c}\mathrm{Y} \\
(\mathrm{E}, 6 \text { mo prior) } \\
\end{array}$ & \\
\hline Cholestatic hepatitis [31] & NR & $35 \mathrm{y} / \mathrm{o} \mathrm{F}$ & $\mathrm{PO}$ & $\mathrm{N}$ & $\mathrm{N}$ & $\mathrm{N}$ & \\
\hline Cholestatic hepatitis [31] & 49 days & $17 \mathrm{y} / \mathrm{oF}$ & $\mathrm{PO}$ & $\mathrm{N}$ & $\mathrm{N}$ & $\mathrm{N}$ & \\
\hline Cholestatic hepatitis [31] & 7 days & $54 \mathrm{y} / \mathrm{o} \mathrm{F}$ & $\mathrm{PO}$ & $\mathrm{N}$ & $\mathrm{N}$ & $\mathrm{N}$ & \\
\hline Cholestatic hepatitis [31] & 1 day & $22 \mathrm{y} / \mathrm{o} \mathrm{F}$ & $\mathrm{IV}, \mathrm{PO}$ & $\mathrm{N}$ & $\mathrm{N}$ & $\mathrm{N}$ & \\
\hline Cholestatic hepatitis [31] & NR & $19 \mathrm{y} / \mathrm{o} \mathrm{M}$ & $\mathrm{PO}$ & $\mathrm{N}$ & $\mathrm{N}$ & $\begin{array}{c}\mathrm{Y} \\
\text { (E, unknown time) }\end{array}$ & \\
\hline Cholestatic hepatitis [31] & 7 days & $13 \mathrm{y} / \mathrm{o} \mathrm{F}$ & $\mathrm{PO}$ & $\mathrm{N}$ & $\mathrm{N}$ & $\mathrm{N}$ & \\
\hline MPR [32] & 8 days & $59 \mathrm{y} / \mathrm{oF}$ & $\mathrm{PO}$ & $\mathrm{N}$ & $\mathrm{N}$ & $\mathrm{N}$ & \\
\hline
\end{tabular}


Table 1. Cont.

\begin{tabular}{|c|c|c|c|c|c|c|c|}
\hline Reaction & Time of Onset ${ }^{\#}$ & Demographics & Dosage Form & Concomitant Agents & Allergy Evaluation/Confirmation & Prior Sensitization & Notes \\
\hline SJS [33] & 7days & $8 \mathrm{y} / \mathrm{o} \mathrm{M}$ & $\mathrm{PO}$ & $\mathrm{N}$ & $\mathrm{N}$ & $\mathrm{N}$ & Fatal \\
\hline Hepatotoxicity [34] & Several h & $23 \mathrm{y} / \mathrm{o} \mathrm{M}$ & $\mathrm{PO}$ & $\mathrm{N}$ & $\mathrm{N}$ & $\begin{array}{c}\mathrm{Y} \\
(\mathrm{E}, 13 \mathrm{yrs} \text { prior }) \\
\end{array}$ & \\
\hline Hepatotoxicity [34] & Several $\mathrm{h}$ & $71 \mathrm{y} / \mathrm{o} \mathrm{F}$ & $\mathrm{PO}$ & $\mathrm{N}$ & $\mathrm{N}$ & $\begin{array}{c}\mathrm{Y} \\
(\mathrm{E}, 15 \mathrm{yrs} \text { prior })\end{array}$ & \\
\hline $\mathrm{CD}[35]$ & NR & $72 \mathrm{y} / \mathrm{o} \mathrm{F}$ & TOP & $\mathrm{N}$ & $\begin{array}{c}\mathrm{Y} \\
(\mathrm{PT}+) \\
\end{array}$ & $\begin{array}{c}\mathrm{Y} \\
(\mathrm{E}, 2 \text { yrs prior) } \\
\end{array}$ & \\
\hline Interstitial Nephritis [36] & Several wks & $39 \mathrm{y} / \mathrm{o} \mathrm{F}$ & $\mathrm{PO}$ & $\mathrm{N}$ & $\begin{array}{c}\mathrm{Y} \\
(\mathrm{MMIT}+)\end{array}$ & $\mathrm{N}$ & \\
\hline Rash [37] & 9 days & NR & $\mathrm{PO}$ & $\mathrm{N}$ & $\mathrm{N}$ & $\mathrm{N}$ & \\
\hline Cholestatic hepatitis [38] & 2 days & $46 \mathrm{y} / \mathrm{o} \mathrm{F}$ & $\mathrm{PO}$ & $\mathrm{N}$ & $\mathrm{N}$ & $\begin{array}{c}\mathrm{Y} \\
\text { (E, twice in last } 2 \text { mo.) }\end{array}$ & \\
\hline Cholestatic hepatitis [38] & 6 days & $53 \mathrm{y} / \mathrm{o} \mathrm{F}$ & $\mathrm{PO}$ & $\mathrm{N}$ & $\mathrm{N}$ & $\begin{array}{c}\mathrm{Y} \\
\text { (E, twice in last } 2 \text { mo.) }\end{array}$ & \\
\hline FDE [39] & 3 days & $46 \mathrm{y} / \mathrm{o} \mathrm{M}$ & $\mathrm{PO}$ & $\mathrm{N}$ & $\begin{array}{c}\mathrm{Y} \\
(\mathrm{PT}-, \mathrm{DPT}+) \\
\end{array}$ & $\begin{array}{c}\mathrm{Y} \\
\text { (E, yrs prior) } \\
\end{array}$ & \\
\hline TEN [40] & $4-5 \mathrm{~h}$ & $20 \mathrm{y} / \mathrm{o} \mathrm{F}$ & $\mathrm{PO}$ & $\mathrm{N}$ & $\begin{array}{c}\mathrm{Y} \\
(\mathrm{PT}+, \mathrm{DPT}+)\end{array}$ & $\mathrm{N}$ & \\
\hline $\begin{array}{l}\text { Granulomatous } \\
\text { interstitial nephritis [41] }\end{array}$ & 5 days & $47 \mathrm{y} / \mathrm{o} \mathrm{M}$ & $\mathrm{PO}$ & $\begin{array}{c}\mathrm{Y} \\
\begin{array}{c}\text { (staggered phenylpropanolamine } \\
\text { and amoxicillin) }\end{array} \\
\end{array}$ & $\mathrm{N}$ & $\mathrm{N}$ & \\
\hline Cholestatic hepatitis [42] & 14 days & $67 \mathrm{y} / \mathrm{oW}$ & $\mathrm{PO}$ & $\mathrm{N}$ & $\mathrm{N}$ & $\mathrm{N}$ & \\
\hline MPE [43] & 1 day & $23 \mathrm{y} / \mathrm{o} \mathrm{M}$ & $\mathrm{PO}$ & $\mathrm{N}$ & $\mathrm{N}$ & $\mathrm{N}$ & $\begin{array}{l}\text { Coninfected with } \\
\text { mononucleosis }\end{array}$ \\
\hline TEN [44] & 2 days & $4 \mathrm{y} / \mathrm{o} \mathrm{M}$ & $\mathrm{PO}$ & $\begin{array}{c}\mathrm{Y} \\
\text { (sulfisoxazole) }\end{array}$ & $\begin{array}{c}\mathrm{Y} \\
(\mathrm{DPT}+)\end{array}$ & Y (15 days prior) & Slow aceylator \\
\hline FDE [45] & NR & $46 \mathrm{y} / \mathrm{o} \mathrm{M}$ & $\mathrm{PO}$ & $\begin{array}{c}\mathrm{Y} \\
\text { (sulfamide, pyrazolone) }\end{array}$ & $\begin{array}{c}\mathrm{Y} \\
\text { (E, PT+, DPT+; sulfa DPT--, } \\
\text { magnesium dipirona DPT-) }\end{array}$ & $\begin{array}{c}\mathrm{Y} \\
\text { (E, unknown time) }\end{array}$ & \\
\hline MAS [46] & NR & $\begin{array}{l}\mathrm{n}=26,<18 \mathrm{y} / \mathrm{o} \\
\text { and gender NR }\end{array}$ & $\mathrm{PO}$ & $\begin{array}{c}\mathrm{Y} \\
\text { (sulfisoxazole) }\end{array}$ & $\mathrm{Y}(\mathrm{PT}+, \mathrm{DPT}+)$ & NR & \\
\hline MPE [47] & $5 \min$ & $20 \mathrm{y} / \mathrm{o} \mathrm{F}$ & $\mathrm{PO}$ & $\mathrm{N}$ & Y (SPT-, PT- DPT+) & $\begin{array}{c}\mathrm{Y} \\
\text { (same reaction with spiramycin 1 yr prior) }\end{array}$ & \\
\hline FDE [48] & 2 and 4 days & $27 \mathrm{y} / \mathrm{o} \mathrm{M}$ & $\mathrm{PO}$ & $\mathrm{N}$ & $\begin{array}{c}\mathrm{Y} \\
(\mathrm{DPT}+)\end{array}$ & $\begin{array}{c}\mathrm{Y} \\
\text { (E, same reaction months apart) }\end{array}$ & \\
\hline $\begin{array}{l}\text { MPE, pruritus, fever, } \\
\text { hepatitis [49] }\end{array}$ & 2 days & $38 \mathrm{y} / \mathrm{o} \mathrm{F}$ & $\mathrm{PO}$ & $\mathrm{N}$ & $\mathrm{N}$ & $\mathrm{N}$ & \\
\hline $\begin{array}{l}\text { Urticaria, palmar } \\
\text { pruritus [50] }\end{array}$ & $10 \mathrm{~min}$ & $25 \mathrm{y} / \mathrm{o} \mathrm{F}$ & $\mathrm{PO}$ & $\mathrm{N}$ & $\begin{array}{c}\mathrm{Y} \\
(\mathrm{SPT}+\mathrm{PT}-, \mathrm{DPT}+, \mathrm{HRT}+)\end{array}$ & $\begin{array}{c}\mathrm{Y} \\
(\mathrm{E} \text {, same reaction } 92 \text { days apart) }\end{array}$ & \\
\hline $\mathrm{CD}[51]$ & Several days & $46 \mathrm{y} / \mathrm{o} \mathrm{M}$ & TOP & $\mathrm{N}$ & $\begin{array}{c}\mathrm{Y} \\
(\mathrm{PT}+)\end{array}$ & $\begin{array}{c}\mathrm{Y} \\
\text { (received top and PO sequentially) }\end{array}$ & \\
\hline
\end{tabular}


Table 1. Cont.

\begin{tabular}{|c|c|c|c|c|c|c|c|}
\hline Reaction & Time of Onset ${ }^{\#}$ & Demographics & Dosage Form & Concomitant Agents & Allergy Evaluation/Confirmation & Prior Sensitization & Notes \\
\hline $\begin{array}{l}\text { Generalized } \\
\text { dermatitis [51] }\end{array}$ & $<1$ day & $46 \mathrm{y} / \mathrm{o} \mathrm{M}$ & $\mathrm{PO}$ & $\mathrm{N}$ & $\begin{array}{c}\mathrm{Y} \\
(\mathrm{PT}+)\end{array}$ & $\begin{array}{c}\mathrm{Y} \\
\text { (received top and PO sequentially) }\end{array}$ & \\
\hline SJS [52] & $16 \mathrm{~h}$ & $64 \mathrm{y} / \mathrm{o} \mathrm{M}$ & $\mathrm{PO}$ & $\mathrm{N}$ & $\mathrm{N}$ & $\mathrm{N}$ & \\
\hline Urticaria [53] & $30 \mathrm{~min}$ & $27 \mathrm{y} / \mathrm{o} \mathrm{F}$ & $\mathrm{PO}$ & $\mathrm{N}$ & $\begin{array}{c}\mathrm{Y} \\
(\mathrm{SPT}+, \mathrm{DPT}+)\end{array}$ & $\begin{array}{c}\mathrm{Y} \\
\text { (same reaction } 2 \text { yrs apart) }\end{array}$ & Serum IgE+ \\
\hline AGEP [54] & 2 days & $46 \mathrm{y} / \mathrm{o} \mathrm{F}$ & $\mathrm{PO}$ & $\mathrm{N}$ & $\begin{array}{c}\mathrm{Y} \\
(\mathrm{PT}+)\end{array}$ & $\begin{array}{c}\mathrm{Y} \\
(\mathrm{S}, 2 \text { days apart }) \\
\end{array}$ & $\begin{array}{l}\text { Cross reactivity } \\
\text { with } E \text { and S }\end{array}$ \\
\hline SJS [55] & $<24 \mathrm{~h}$ & $23 \mathrm{y} / \mathrm{oF}$ & $\mathrm{PO}$ & $\begin{array}{c}\text { Y } \\
\text { (chlorpheniramine, } \\
\text { pseudoephedrine, naphazoline) }\end{array}$ & $\mathrm{N}$ & Unknown & \\
\hline $\mathrm{CD}[56]$ & 8 days & $21 \mathrm{y} / \mathrm{o} \mathrm{F}$ & TOP & $\mathrm{N}$ & $\begin{array}{c}\mathrm{Y} \\
(\mathrm{PT}+)\end{array}$ & $\begin{array}{c}\mathrm{Y} \\
\text { (TOP E multiple times without reaction) }\end{array}$ & \\
\hline Cholestatic hepatitis [57] & 4 days; 5 days & $38 \mathrm{y} / 0 \mathrm{~F}$ & $\mathrm{PO}$ & $\mathrm{N}$ & $\begin{array}{c}\mathrm{Y} \\
(\mathrm{DPT}+)\end{array}$ & $\begin{array}{c}\mathrm{Y} \\
\text { (E, same reaction } 1.5 \text { yrs apart) }\end{array}$ & \\
\hline CD [58] & 12 days & $35 \mathrm{y} / \mathrm{o} \mathrm{M}$ & TOP & $\begin{array}{c}\mathrm{Y} \\
\text { (metronidazole PO and TOP) }\end{array}$ & $\begin{array}{c}\mathrm{Y} \\
(\mathrm{E}, \mathrm{PT}+; \text { metronidazole PT-) }\end{array}$ & $\mathrm{N}$ & \\
\hline Anaphylaxis [59] & $30 \mathrm{~min}$ & $24 \mathrm{y} / \mathrm{o} \mathrm{M}$ & $\mathrm{PO}$ & $\mathrm{N}$ & $\begin{array}{c}\mathrm{Y} \\
(\mathrm{SPT}-, \mathrm{IDT}-\mathrm{DPT}+)\end{array}$ & $\mathrm{N}$ & \\
\hline LCV [60] & Several hours & $1.5 \mathrm{y} / \mathrm{o} \mathrm{M}$ & $\mathrm{PO}$ & $\mathrm{N}$ & $\begin{array}{c}\mathrm{Y} \\
(\mathrm{LTT}+)\end{array}$ & $\begin{array}{c}\mathrm{Y} \\
\text { (E unknown time) }\end{array}$ & Declined PT \\
\hline $\begin{array}{l}\text { Hypersensitivity } \\
\text { unspecified [61] }\end{array}$ & NR & $\begin{array}{c}N=5 \\
4 \mathrm{M}, 1 \mathrm{~F} ; 3-60 \mathrm{y} / \mathrm{o}\end{array}$ & NR & NR & $\begin{array}{c}\mathrm{Y} \\
(\mathrm{E}, \mathrm{n}=3 \mathrm{SPT}+, \mathrm{n}=5 \mathrm{DPT}+; \\
\mathrm{C} \text { and A SPT- } \text { and DPT-) }\end{array}$ & NR & $\begin{array}{l}\text { No cross-reactivity } \\
\text { with } E, A, C\end{array}$ \\
\hline Pustulosis [62] & 4 days & $23 \mathrm{y} / \mathrm{o} \mathrm{M}$ & $\mathrm{PO}$ & $\mathrm{N}$ & $\begin{array}{c}\mathrm{Y} \\
\text { (E: SPT-, PT-, DPT+; C: DPT-) }\end{array}$ & $\mathrm{N}$ & $\begin{array}{l}\text { Cross reactivity } \\
\text { with E and C }\end{array}$ \\
\hline Urticaria [63] & NR & NR & $\mathrm{PO}$ & NR & $\begin{array}{c}\mathrm{Y} \\
(\mathrm{IDT}+)\end{array}$ & NR & \\
\hline MPR [63] & NR & NR & $\mathrm{PO}$ & NR & $\begin{array}{c}\mathrm{Y} \\
(\mathrm{SPT}+)\end{array}$ & NR & \\
\hline FDE [64] & NR & $0-90 \mathrm{y} / \mathrm{o} \mathrm{M}$ or $\mathrm{F}$ & NR & NR & $\begin{array}{c}\mathrm{Y} \\
(\mathrm{n}=6, \mathrm{DPT}+)\end{array}$ & & \\
\hline Cholestatic hepatitis [65] & 10 days & $30 \mathrm{y} / 0 \mathrm{~F}$ & $\mathrm{PO}$ & $\mathrm{N}$ & $\begin{array}{c}\mathrm{Y} \\
(\mathrm{DPT}+)\end{array}$ & $\begin{array}{c}\mathrm{Y} \\
\text { (E, same reaction } 2 \text { yrs prior) }\end{array}$ & \\
\hline $\begin{array}{l}\text { Anaphylaxis (Urticaria } \\
\text { and angioedema [66] }\end{array}$ & Several hours & $39 \mathrm{y} / \mathrm{o} \mathrm{M}$ & $\mathrm{PO}$ & NR & $\mathrm{N}$ & $\begin{array}{c}\mathrm{Y} \\
\text { (E, unknown time) }\end{array}$ & \\
\hline SJS [67] & $16-24 \mathrm{~h}$ & $31 \mathrm{y} / \mathrm{oF}$ & $\mathrm{PO}$ & $\mathrm{N}$ & $\mathrm{N}$ & $\mathrm{N}$ & \\
\hline Cutaneous ADR [68] & NR & $\begin{array}{l}\mathrm{n}=6 \text {, age and } \\
\text { gender NR }\end{array}$ & NR & NR & NR & NR & \\
\hline SJS [69] & 2 days & $20 \mathrm{y} / \mathrm{o} \mathrm{M}$ & NR & $\begin{array}{c}\mathrm{Y} \\
\text { (APAP, decongestant) }\end{array}$ & $\mathrm{N}$ & NR & \\
\hline
\end{tabular}


Table 1. Cont

\begin{tabular}{|c|c|c|c|c|c|c|c|}
\hline Reaction & Time of Onset $\#$ & Demographics & Dosage Form & Concomitant Agents & Allergy Evaluation/Confirmation & Prior Sensitization & Notes \\
\hline FDE [70] & $6 \mathrm{~h}$ & $64 \mathrm{y} / \mathrm{o} \mathrm{M}$ & PO & $\mathrm{N}$ & $\begin{array}{c}\mathrm{Y} \\
(\mathrm{E} \text { and } \mathrm{C}, \mathrm{PT}+)\end{array}$ & $\begin{array}{c}\mathrm{Y} \\
\text { (E, two prior times with same reaction) }\end{array}$ & $\begin{array}{l}\text { Cross reactivity } \\
\text { with E and C }\end{array}$ \\
\hline EM, SJS or TEN [71] & NR & $\begin{array}{c}\mathrm{n}=4,(<18 \mathrm{y} / \mathrm{o} \text { and } \\
\text { gender NR })\end{array}$ & NR & NR & NR & NR & \\
\hline Urticaria [72] & NR & $\begin{array}{c}\mathrm{n}=3,<15 \mathrm{y} / \mathrm{o} \text { and } \\
\text { gender NR }\end{array}$ & NR & NR & $\mathrm{N}$ & NR & \\
\hline FDE [72] & NR & $\begin{array}{l}<15 \mathrm{y} / \mathrm{o} \text { and } \\
\text { gender NR }\end{array}$ & NR & NR & $\mathrm{N}$ & NR & \\
\hline MPR [72] & NR & $\begin{array}{l}<15 \mathrm{y} / \mathrm{o} \text { and } \\
\text { gender NR }\end{array}$ & NR & NR & $\mathrm{N}$ & NR & \\
\hline MPR [73] & NIR & $\begin{array}{l}<18 \mathrm{y} / \mathrm{o} \text { and } \\
\text { gender NR }\end{array}$ & NR & NR & $\begin{array}{c}\mathrm{Y} \\
(\mathrm{n}=3, \mathrm{SPT}-, \mathrm{DPT}-)\end{array}$ & NR & \\
\hline Anaphylaxis [74] & NR & $68 \mathrm{y} / \mathrm{o} \mathrm{F}$ & NR & NR & $\mathrm{N}$ & $Y(R)$ & Desensitized (C \\
\hline Exanthema [75] & $12 \mathrm{~h}$ & $\begin{array}{c}19 \mathrm{y} / \mathrm{o} \\
\text { gender NR }\end{array}$ & $\mathrm{PO}$ & $\mathrm{N}$ & $\begin{array}{c}\text { Y } \\
(\text { IDT-, PT-, SPT-, ODT+) }\end{array}$ & $\mathrm{N}$ & \\
\hline Exanthema [75] & $8 \mathrm{~h}$ & $\begin{array}{c}22 \mathrm{y} / \mathrm{o} \\
\text { gender NR }\end{array}$ & $\mathrm{PO}$ & $\mathrm{N}$ & $\begin{array}{c}\mathrm{Y} \\
\text { (IDT-, PT-, SPT-, ODT+) }\end{array}$ & $\mathrm{N}$ & \\
\hline AGEP [76] & 3 days & $61 \mathrm{y} / \mathrm{o} \mathrm{M}$ & $\mathrm{PO}$ & $\begin{array}{c}\mathrm{Y} \\
\text { (fluconazole) }\end{array}$ & $\mathrm{N}$ & NR & \\
\hline Urticaria [77] & NR & $\mathrm{n}=1(\geq 17-79 \mathrm{y} / \mathrm{o})$ & NR & NR & $\begin{array}{c}\mathrm{Y} \\
(\mathrm{DPT}+)\end{array}$ & NR & \\
\hline
\end{tabular}

Abbreviations: $\mathrm{ACDR}=$ acute cutaneous drug reaction ${ }^{\mathrm{a}} ; \mathrm{ADR}=$ adverse drug reaction; $\mathrm{AGEP}=$ acute generalized exanthematous pustulosis; $\mathrm{APAP}=$ acetaminophen; $\mathrm{ASA}=$ aspirin; $\mathrm{A}=$ azithromycin; $\mathrm{C}=$ clarithromycin; $\mathrm{CD}=$ contact dermatitis; $\mathrm{DR}=$ delayed reaction; $\mathrm{DPT}=$ drug provocation test; DRESS = Drug reaction with eosinophilia and systemic symptoms syndrome; $\mathrm{E}=$ erythromycin; $\mathrm{EM}=$ erythema multiforme; $\mathrm{ER}=$ extended release; $\mathrm{ETH}=$ ethambutol; $\mathrm{F}=$ female; $\mathrm{FDX}=$ fidaxomicin; $\mathrm{HRT}=$ leukocyte histamine release test; HSP = Henoch-Schönlein Purpura; $\mathrm{h}=$ hour(s); HCTZ = hydrochlorothiazide; HIV = human immunodeficiency virus; IBU = ibuprofen; IDT = intradermal test; Ig E = immunoglobulin $\mathrm{E} ; \mathrm{IM}=$ intramuscular; $\mathrm{IR}=$ immediate reaction $(<1 \mathrm{~h})$; LABD = Linear Immunoglobulin A Bullous Dermatosis; $\mathrm{LCV}=$ leukocytoclastic vasculitis; $\mathrm{LTT}=$ lymphocyte transformation test; MAS = multiple allergy sensitivity; MDT = mast cell degranulation test; min= minutes; mo = months; MMIT = macrophage migration inhibitory test; MPE = maculopapular exanthema; $\mathrm{MPR}=$ maculopapular rash; $\mathrm{N}=$ no; $\mathrm{NIR}=$ nonimmediate reaction $(1-72 \mathrm{~h})$; $\mathrm{NR}=$ not reported; $\mathrm{NTG}=$ nitroglycerin; opth = ophthalmic; $\mathrm{PT}=$ patch test; $\mathrm{R}=$ roxithromycin; $\mathrm{RIF}=$ rifampin; RFB = rifabutin; $\mathrm{S}=$ spiramycin; $\mathrm{SJS}=$ Steven's Johnson syndrome; $\mathrm{SOB}=$ shortness of breath; $\mathrm{SPT}=$ skin prick test; $\mathrm{ST}=\mathrm{scratch}$ test; $\mathrm{TEN}=$ toxic epidermal necrolysis; $\mathrm{TMP} / \mathrm{SMX}=$ trimethoprim/sulfamethoxazole; top = topical; $\mathrm{wks}=$ weeks; $\mathrm{Y}=$ yes; yrs = years; $\mathrm{y} / \mathrm{o}=$ years old. ${ }^{*}$ Exact time course unknown as awoke with FDE. ${ }^{\#}$ Time of onset means time when symptoms began to occur either during macrolide therapy or after completion of macrolide therapy*ACDR defined as allergic reaction, adverse drug reaction, pruritis, general swelling, local or general redness, erythema, rash, urticaria, or other skin disease. ${ }^{\pi}$ Review was meant to include azithromycin, clarithromycin, erythromycin, and fidaxomicin; however, there is a chance these data include excluded macrolides since the type of macrolides reported were not fully specified 
Table 2. Summary of published literature reporting clarithromycin hypersensitivity.

\begin{tabular}{|c|c|c|c|c|c|c|c|}
\hline Reaction & Time of Onset ${ }^{\#}$ & Demographics & Dosage Form & Concomitant Agents & Allergy Evaluation/Confirmation & Prior Sensitization & Notes \\
\hline Urticaria [72] & NR & $\begin{array}{c}\mathrm{n}=1,<15 \mathrm{y} / \mathrm{o} \text { and } \\
\text { gender } \mathrm{NR}\end{array}$ & NR & NR & $\mathrm{N}$ & NR & \\
\hline MPR [72] & NR & $\begin{array}{c}\mathrm{n}=1,<15 \mathrm{y} / \mathrm{o} \text { and } \\
\text { gender NR }\end{array}$ & NR & NR & $\mathrm{N}$ & NR & \\
\hline $\begin{array}{l}\text { Anaphylaxis (urticaria, } \\
\text { angioedema) [73] }\end{array}$ & NIR & $\begin{array}{l}<18 \mathrm{y} / \mathrm{o} \text { and } \\
\text { gender NR }\end{array}$ & NR & NR & $\begin{array}{c}\mathrm{Y} \\
\text { (SPT-, DPT-) }\end{array}$ & NR & \\
\hline MPR [73] & NIR & $\begin{array}{l}<18 \mathrm{y} / \mathrm{o} \text { and } \\
\text { gender NR }\end{array}$ & NR & NR & $\begin{array}{c}\mathrm{Y} \\
(\mathrm{SPT}-, \text { DPT-) }\end{array}$ & NR & \\
\hline FDE [75] & $6 \mathrm{~h}$ & $\begin{array}{l}37 \mathrm{y} / \mathrm{o} \text { and } \\
\text { gender NR }\end{array}$ & $\mathrm{PO}$ & NR & $\begin{array}{c}\mathrm{Y} \\
(\mathrm{IDT}-, \mathrm{PT}-, \text { SPT-, ODT+) }\end{array}$ & NR & \\
\hline Exanthema [75] & $20 \mathrm{~h}$ & $\begin{array}{l}50 \mathrm{y} / \mathrm{o} \text { and } \\
\text { gender NR }\end{array}$ & $\mathrm{PO}$ & NR & $\begin{array}{c}\mathrm{Y} \\
(\mathrm{IDT}-, \mathrm{PT}-, \mathrm{SPT}-, \mathrm{ODT}+)\end{array}$ & NR & \\
\hline Thrombocytopenia [78] & 2 weeks & $30 \mathrm{y} / \mathrm{o} \mathrm{M}$ & $\mathrm{PO}$ & $\begin{array}{c}\mathrm{Y} \\
\text { (amikacin, clofazimine) }\end{array}$ & $\mathrm{N}$ & $\mathrm{N}$ & \\
\hline LCV [79] & 1 day & $68 \mathrm{y} / \mathrm{om}$ & $\mathrm{PO}$ & $\mathrm{N}$ & $\mathrm{N}$ & $\mathrm{N}$ & \\
\hline $\begin{array}{l}\text { Thrombocytopenic } \\
\text { purpura [80] }\end{array}$ & 7 days & $74 \mathrm{y} / \mathrm{o} \mathrm{M}$ & $\mathrm{PO}$ & $\begin{array}{c}\mathrm{Y} \\
\text { (digoxin) }\end{array}$ & $\mathrm{N}$ & $\mathrm{N}$ & \\
\hline Angioedema [81] & $2 \mathrm{~h}$ & $92 \mathrm{y} / \mathrm{o} \mathrm{F}$ & $\mathrm{PO}$ & $\begin{array}{c}\mathrm{Y} \\
\text { (NTG, ASA, digoxin, captopril) }\end{array}$ & $\mathrm{N}$ & $\begin{array}{c}\mathrm{Y} \\
\text { (6 days prior) }\end{array}$ & \\
\hline MPR and angioedema [82] & $8 \mathrm{~h}$ & $25 \mathrm{y} / \mathrm{o} \mathrm{F}$ & $\mathrm{PO}$ & $\mathrm{N}$ & $\begin{array}{c}\mathrm{Y} \\
(\mathrm{SPT}-, \mathrm{PT}-, \mathrm{DPT}+)\end{array}$ & $\begin{array}{c}\mathrm{Y} \\
\text { (unknown time) }\end{array}$ & \\
\hline FDE [83] & 3 days & $58 \mathrm{y} / \mathrm{o} \mathrm{M}$ & $\mathrm{PO}$ & $\mathrm{N}$ & $\mathrm{Y}(\mathrm{PT}+)$ & $\mathrm{N}$ & \\
\hline LCV [84] & 6 days & $83 \mathrm{y} / \mathrm{oF}$ & $\mathrm{PO}$ & $\begin{array}{c}\mathrm{Y} \\
\text { (diltiazem ER, ASA, } \\
\text { triamterene/HCTZ) }\end{array}$ & $\mathrm{N}$ & $\mathrm{N}$ & \\
\hline HSP [85] & 10 days & $25 \mathrm{y} / \mathrm{oM}$ & $\mathrm{PO}$ & $\mathrm{N}$ & $\mathrm{N}$ & $\mathrm{N}$ & \\
\hline FDE [86] & 3 days & $83 \mathrm{y} / \mathrm{oF}$ & $\mathrm{PO}$ & $\begin{array}{l}\mathrm{Y} \\
\text { (carbosistein, salicylamide, APAP, } \\
\text { caffeine, promethazine } \\
\text { methylenedisalicilate) }\end{array}$ & $\begin{array}{c}\mathrm{Y} \\
(\mathrm{PT}-, \text { DPT+) }\end{array}$ & $\mathrm{N}$ & \\
\hline $\begin{array}{l}\text { Dyspnea, bronchospasm, } \\
\text { cough [87] }\end{array}$ & Min & $44 \mathrm{y} / \mathrm{oF}$ & $\mathrm{PO}$ & $\mathrm{N}$ & $\begin{array}{c}\mathrm{Y} \\
(\mathrm{DPT}+)\end{array}$ & $\mathrm{N}$ & \\
\hline Hepatitis and TEN [88] & 7 days & $47 \mathrm{y} / \mathrm{o} \mathrm{M}$ & $\mathrm{PO}$ & $\begin{array}{c}\mathrm{Y} \\
\text { (disulfiram, APAP) }\end{array}$ & $\mathrm{N}$ & $\mathrm{N}$ & Fatal \\
\hline
\end{tabular}


Table 2. Cont

\begin{tabular}{|c|c|c|c|c|c|c|c|}
\hline Reaction & Time of Onset ${ }^{\#}$ & Demographics & Dosage Form & Concomitant Agents & Allergy Evaluation/Confirmation & Prior Sensitization & Notes \\
\hline HSP [89] & 4 days & $48 \mathrm{y} / \mathrm{o} \mathrm{M}$ & $\mathrm{PO}$ & $\mathrm{N}$ & $\mathrm{N}$ & $\mathrm{N}$ & \\
\hline Pulmonary infiltrates [90] & $\begin{array}{l}3 \text { days (episode } 1 \& 3 \text { ) } \\
12 \text { days (episode } 2 \text { ) }\end{array}$ & $17 \mathrm{y} / \mathrm{o} \mathrm{M}$ & $\mathrm{PO}$ & $\begin{array}{c}\mathrm{Y} \\
\text { (cefotaxime episode 2, } \\
\text { prednisone episode 3) }\end{array}$ & $\begin{array}{c}\mathrm{Y} \\
\text { (DPT+) }\end{array}$ & $\begin{array}{c}\mathrm{Y} \\
\text { (same reaction } 3 \text { times) }\end{array}$ & \\
\hline TEN [91] & 3 days & $65 \mathrm{y} / \mathrm{oW}$ & $\mathrm{PO}$ & NR & $\mathrm{N}$ & $\mathrm{N}$ & Fatal \\
\hline $\begin{array}{c}\text { Eosinophilic } \\
\text { pneumonia [92] }\end{array}$ & 3 days & $74 \mathrm{y} / \mathrm{o} \mathrm{M}$ & $\mathrm{PO}$ & NR & $\begin{array}{c}\mathrm{Y} \\
(\mathrm{LTT}-, \text { DPT +) } \\
\end{array}$ & $\mathrm{N}$ & \\
\hline FDE [93] & 4 days & $68 \mathrm{y} / \mathrm{o} \mathrm{F}$ & $\mathrm{PO}$ & NR & $\begin{array}{c}\mathrm{Y} \\
(\mathrm{PT}-, \mathrm{DPT}+)\end{array}$ & $\mathrm{N}$ & \\
\hline TEN [94] & 2 days & $29 \mathrm{y} / \mathrm{o} \mathrm{F}$ & $\mathrm{PO}$ & APAP, ASA and erdosteine & $\mathrm{N}$ & $\mathrm{N}$ & \\
\hline SJS [95] & NR & $\begin{array}{l}<18 \mathrm{y} / \mathrm{o} \text { and } \\
\text { gender NR }\end{array}$ & PO & IBU & $\mathrm{N}$ & $\mathrm{N}$ & \\
\hline TEN [96] & 2 days & $2 \mathrm{y} / \mathrm{o}$ & $\mathrm{PO}$ & $\mathrm{N}$ & $\mathrm{N}$ & $\mathrm{N}$ & \\
\hline ADRs [97] & NR & $\begin{array}{c}\mathrm{n}=1(<18 \mathrm{y} / \mathrm{o} \text { and } \\
\text { gender NR) }\end{array}$ & NR & NR & $\begin{array}{c}\mathrm{Y} \\
(\mathrm{NR})\end{array}$ & NR & \\
\hline Anaphylaxis [98] & 4 days & $4 \mathrm{y} / \mathrm{oF}$ & $\mathrm{PO}$ & $\begin{array}{c}\mathrm{Y} \\
\text { (fluticasone/salmeterol) }\end{array}$ & $\mathrm{N}$ & $\begin{array}{c}\mathrm{Y} \\
\text { (unknown time) }\end{array}$ & \\
\hline Urticaria [99] & $10 \mathrm{~min}$ & $\begin{array}{l}(<15 \mathrm{y} / \mathrm{o} \text { and } \\
\text { gender NR })\end{array}$ & $\mathrm{PO}$ & $\mathrm{N}$ & $\begin{array}{c}\mathrm{Y} \\
(\mathrm{SPT}-, \mathrm{IDT}+, \mathrm{DPT}+)\end{array}$ & NR & \\
\hline Urticaria, angioedema [99] & $20 \mathrm{~min}$ & $\begin{array}{l}(<15 \mathrm{y} / \mathrm{o} \text { and } \\
\text { gender NR })\end{array}$ & $\mathrm{PO}$ & $\mathrm{N}$ & $\begin{array}{c}\mathrm{Y} \\
(\mathrm{SPT}-, \mathrm{IDT}+, \mathrm{DPT}+)\end{array}$ & NR & \\
\hline MPE, pruritis [99] & 3 days & $\begin{array}{l}(<15 \mathrm{y} / \mathrm{o} \text { and } \\
\text { gender NR })\end{array}$ & $\mathrm{PO}$ & $\mathrm{N}$ & $\begin{array}{c}\mathrm{Y} \\
(\mathrm{SPT}-, \mathrm{IDT}+, \mathrm{DPT}+)\end{array}$ & NR & \\
\hline MPE, pruritis [99] & 4 days & $\begin{array}{l}(<15 \mathrm{y} / \mathrm{o} \text { and } \\
\text { gender NR })\end{array}$ & $\mathrm{PO}$ & $\mathrm{N}$ & $\begin{array}{c}\mathrm{Y} \\
(\mathrm{SPT}-, \mathrm{IDT}+, \mathrm{DPT}+)\end{array}$ & NR & \\
\hline Urticaria, dyspnea [100] & 6 days & $68 \mathrm{y} / \mathrm{o} \mathrm{F}$ & $\mathrm{PO}$ & $\mathrm{N}$ & $\begin{array}{c}\mathrm{Y} \\
(\mathrm{SPT}-, \mathrm{IDT}+)\end{array}$ & $\begin{array}{c}\mathrm{Y} \\
\text { (C, 6 mo. prior without reaction) }\end{array}$ & Desensitized with $C$ \\
\hline SJS [101] & 3-4 days & $\begin{array}{l}>18 \mathrm{y} / \mathrm{o} \text { and } \\
\text { gender NR }\end{array}$ & $\mathrm{PO}$ & $\begin{array}{c}\mathrm{Y} \\
\text { (RFB, TMP/SMX, } \\
\text { Hidup herbal tea) }\end{array}$ & $\mathrm{N}$ & Unknown & \\
\hline SJS [101] & NR & $\begin{array}{l}>18 \mathrm{y} / \mathrm{o} \text { and } \\
\text { gender NR }\end{array}$ & $\mathrm{PO}$ & $\begin{array}{c}\mathrm{Y} \\
\text { (dapsone, ciprofloxacin) }\end{array}$ & $\mathrm{N}$ & Unknown & \\
\hline
\end{tabular}


Table 2. Cont

\begin{tabular}{|c|c|c|c|c|c|c|c|}
\hline Reaction & Time of Onset \# & Demographics & Dosage Form & Concomitant Agents & Allergy Evaluation/Confirmation & Prior Sensitization & Notes \\
\hline Diffuse urticarial rash [102] & $15-30 \mathrm{~min}$ & $11 \mathrm{y} / \mathrm{o} \mathrm{F}$ & IV & $\begin{array}{c}\mathrm{Y} \\
\text { (amikacin) }\end{array}$ & $\begin{array}{c}\mathrm{Y} \\
\text { (C, SPT-, IDT-, DPT+; } \\
\text { A, SPT-, IDT-) }\end{array}$ & $\mathrm{N}$ & $\begin{array}{c}\text { Cross reactivity with C and A. } \\
\text { Desensitized successfully } \\
\text { with C and unsuccessfully A }\end{array}$ \\
\hline FDE [103] & NR & $30 \mathrm{y} / \mathrm{oM}$ & $\mathrm{PO}$ & $\mathrm{N}$ & $\begin{array}{c}\mathrm{Y} \\
(\mathrm{DPT}+)\end{array}$ & $\begin{array}{c}\mathrm{Y} \\
\text { (same reaction } 4 \text { mo. apart) }\end{array}$ & \\
\hline IR [104] & $<1 \mathrm{~h}$ & $\mathrm{n}=37, \mathrm{NR}$ & $\mathrm{PO}$ & NR & $\begin{array}{c}\mathrm{Y} \\
(\mathrm{n}=2 \mathrm{ST}+) \\
\end{array}$ & NR & \\
\hline NIR [104] & $1-3 \mathrm{~h}$ & $\mathrm{n}=37, \mathrm{NR}$ & $\mathrm{PO}$ & NR & $\begin{array}{c}\mathrm{Y} \\
(\mathrm{n}=2 \mathrm{ST}+, \mathrm{n}=2 \mathrm{DPT}+)\end{array}$ & NR & \\
\hline DRESS [105] & 4 weeks & $79 \mathrm{y} / \mathrm{o} \mathrm{M}$ & $\mathrm{PO}$ & $\begin{array}{c}\text { Y } \\
\text { (RIF, ETH, levothyroxine, } \\
\text { hydrocortisone, tramadol, } \\
\text { lisinopril, APAP, carvedilol) }\end{array}$ & $\mathrm{N}$ & NR & \\
\hline MPE [106] & NR & $14 \mathrm{y} / \mathrm{o} \mathrm{M}$ & $\mathrm{PO}$ & NR & $\begin{array}{c}\mathrm{Y} \\
\text { (SPT-, IDT-, DPT+) }\end{array}$ & $\begin{array}{c}\mathrm{Y} \\
\text { (1 mo. apart and same reaction) }\end{array}$ & \\
\hline Urticaria [106] & NR & $30 \mathrm{y} / \mathrm{o} \mathrm{F}$ & $\mathrm{PO}$ & NR & $\begin{array}{c}\mathrm{Y} \\
\text { (IDT-, DPT+) }\end{array}$ & $\mathrm{N}$ & \\
\hline Anaphylaxis [121] & 2 days & $9 \mathrm{y} / \mathrm{o}$, gender NR & $\mathrm{PO}$ & NR & $\mathrm{N}$ & $\mathrm{N}$ & \\
\hline
\end{tabular}

Abbreviations: $\mathrm{ACDR}=$ acute cutaneous drug reaction ${ }^{\mathrm{a}} ; \mathrm{ADR}=$ adverse drug reaction; $\mathrm{AGEP}=$ acute generalized exanthematous pustulosis; $\mathrm{APAP}=$ acetaminophen; $\mathrm{ASA}=$ aspirin; $\mathrm{A}=$ azithromycin; $\mathrm{C}=$ clarithromycin; $\mathrm{CD}=$ contact dermatitis; $\mathrm{DR}=$ delayed reaction; $\mathrm{DPT}=$ drug provocation test; $\mathrm{DRESS}=\mathrm{Drug}$ reaction with eosinophilia and systemic symptoms syndrome; $\mathrm{E}=$ erythromycin; $\mathrm{EM}=$ erythema multiforme; $\mathrm{ER}=$ extended release; $\mathrm{ETH}=$ ethambutol; F = female; FDX = fidaxomicin; HRT = leukocyte histamine release test; HSP = Henoch-Schönlein Purpura; h = hour(s); HCTZ = hydrochlorothiazide; HIV = human immunodeficiency virus; IBU = ibuprofen; IDT = intradermal test; Ig E = immunoglobulin $\mathrm{E}$; IM = intramuscular; IR = immediate reaction $(<1 \mathrm{~h}) ; \mathrm{LABD}=$ Linear Immunoglobulin A Bullous Dermatosis; LCV = leukocytoclastic vasculitis; LTT = lymphocyte transformation test; MAS = multiple allergy sensitivity; MDT = mast cell degranulation test; min= minutes; mo = months; MMIT = macrophage migration inhibitory test; MPE = maculopapular exanthema; MPR = maculopapular rash; $\mathrm{N}=$ no; NIR= nonimmediate reaction $(1-72 \mathrm{~h}) ; \mathrm{NR}=$ not reported; NTG = nitroglycerin; opth = ophthalmic; PT = patch test; $\mathrm{R}=$ roxithromycin; $\mathrm{RIF}=$ rifampin; RFB = rifabutin; $\mathrm{S}=$ spiramycin; $\mathrm{SJS}=$ Steven's Johnson syndrome; $\mathrm{SOB}=$ shortness of breath; $\mathrm{SPT}=$ skin prick test; $\mathrm{ST}=\mathrm{scratch}$ test; $\mathrm{TEN}=$ toxic epidermal necrolysis; $\mathrm{TMP} / \mathrm{SMX}=$ trimethoprim/sulfamethoxazole; top = topical; $\mathrm{wks}=$ weeks; $\mathrm{Y}=$ yes; yrs = years; $\mathrm{y} / \mathrm{o}=$ years old. * Exact time course unknown as awoke with FDE. \# Time of onset means time when symptoms began to occur either during macrolide therapy or after completion of macrolide therapy. ACDR defined as allergic reaction, adverse drug reaction, pruritis, general swelling, local or general redness, erythema, rash, urticaria, or other skin disease. ${ }^{\pi}$ Review was meant to include azithromycin, clarithromycin, erythromycin, and fidaxomicin; however, there is a chance these data include excluded macrolides since the type of macrolides reported were not fully specified 
Table 3. Summary of published literature reporting azithromycin hypersensitivity.

\begin{tabular}{|c|c|c|c|c|c|c|c|}
\hline Reaction & Time of Onset \# & Demographics & Dosage Form & Concomitant Agents & Allergy Evaluation/Confirmation & Prior Sensitization & Notes \\
\hline TEN [95] & NR & $\begin{array}{c}\mathrm{n}=4,<18 \mathrm{y} / \mathrm{o} \text { and } \\
\text { gender NR }\end{array}$ & $\mathrm{PO}$ & $\begin{array}{c}\mathrm{Y} \\
(\mathrm{IBU})\end{array}$ & $\mathrm{N}$ & $\mathrm{N}$ & \\
\hline EM [95] & NR & $\begin{array}{c}\mathrm{n}=4,<18 \mathrm{y} / \mathrm{o} \text { and } \\
\text { gender NR }\end{array}$ & PO & $\begin{array}{c}\mathrm{Y} \\
(\mathrm{IBU})\end{array}$ & $\mathrm{N}$ & $\mathrm{N}$ & \\
\hline SJS [95] & NR & $\begin{array}{c}\mathrm{n}=4,<18 \mathrm{y} / \mathrm{o} \text { and } \\
\text { gender NR }\end{array}$ & PO & $\begin{array}{c}\mathrm{Y} \\
\text { (IBU) }\end{array}$ & $\mathrm{N}$ & $\mathrm{N}$ & \\
\hline SJS [95] & NR & $\begin{array}{l}<18 \mathrm{y} / \mathrm{o} \text { and } \\
\text { gender NR }\end{array}$ & PO & $\mathrm{N}$ & $\mathrm{N}$ & $\mathrm{N}$ & \\
\hline Anaphylaxis [100] & $2 \mathrm{~h}$ & $68 \mathrm{y} / \mathrm{oF}$ & PO & $\mathrm{N}$ & $\begin{array}{c}\mathrm{Y} \\
(\mathrm{SPT}+, \mathrm{IDT}+)\end{array}$ & $\begin{array}{c}\text { Y } \\
\text { (C,1 day prior } \\
\text { urticaria and dyspnea) }\end{array}$ & $\begin{array}{l}\text { Cross reactivity with } C \text { and } A \text {. } \\
\text { Desensitized with } C \text {. }\end{array}$ \\
\hline IR [104] & $<1 \mathrm{~h}$ & $\mathrm{n}=6, \mathrm{NR}$ & $\mathrm{PO}$ & NR & $\begin{array}{c}\mathrm{Y} \\
(\mathrm{n}=4 \mathrm{ST}+)\end{array}$ & NR & \\
\hline NIR [104] & $1-3 \mathrm{~h}$ & $\mathrm{n}=13, \mathrm{NR}$ & PO & NR & $\begin{array}{c}\mathrm{Y} \\
(\mathrm{n}=5 \mathrm{ST}+, 8 \mathrm{ST}-)\end{array}$ & NR & \\
\hline Toxic pustuloderma [107] & $16 \mathrm{~h}$ & $34 \mathrm{y} / \mathrm{oF}$ & $\mathrm{PO}$ & $\mathrm{N}$ & $\mathrm{N}$ & $\mathrm{N}$ & \\
\hline Angioedema [108] & NR & NR & NR & NR & $\mathrm{N}$ & NR & \\
\hline MPE [109] & 7 days & $20 \mathrm{y} / \mathrm{o} \mathrm{M}$ & $\mathrm{PO}$ & $\mathrm{N}$ & $\mathrm{N}$ & $\mathrm{N}$ & Coinfected with mononucleosis \\
\hline $\begin{array}{l}\text { AMS, fever, generalized } \\
\text { rash, and hepatitis [110] }\end{array}$ & 5 days & $79 \mathrm{y} / \mathrm{o} \mathrm{M}$ & $\mathrm{PO}$ & $\begin{array}{c}\mathrm{Y} \\
\begin{array}{c}\text { (atenolol, benazepril, } \\
\text { clonidine, ASA) }\end{array} \\
\end{array}$ & $\mathrm{N}$ & NR & \\
\hline MPE [111] & $<1$ day & $19 \mathrm{y} / \mathrm{o} \mathrm{M}$ & $\mathrm{PO}$ & $\mathrm{N}$ & $\mathrm{N}$ & $\mathrm{N}$ & Coinfected with mononucleosis \\
\hline Mild pruritus [112] & NR & $\mathrm{n}=135, \mathrm{NR}$ & $\mathrm{PO}$ & NR & $\mathrm{N}$ & NR & \\
\hline Severe pruritus [112] & NR & $\mathrm{n}=19, \mathrm{NR}$ & $\mathrm{PO}$ & NR & $\mathrm{N}$ & NR & \\
\hline Rash [112] & NR & $\mathrm{n}=6, \mathrm{NR}$ & $\mathrm{PO}$ & NR & $\mathrm{N}$ & NR & \\
\hline LCV [113] & 3 days & $8 \mathrm{mo} . \mathrm{M}$ & PO & NR & $\mathrm{N}$ & NS & \\
\hline SJS [114] & 3 days & $5 \mathrm{y} / \mathrm{o} \mathrm{M}$ & PO & $\mathrm{N}$ & $\mathrm{N}$ & $\mathrm{N}$ & \\
\hline FDE [16] & $6 \mathrm{yrs}$ & $\begin{array}{c}\mathrm{n}=2,40 \mathrm{y} / \mathrm{o} \mathrm{M} \\
\text { and } 44 \mathrm{y} / \mathrm{o} \mathrm{M}\end{array}$ & TOP & $\mathrm{N}$ & $\begin{array}{c}\mathrm{Y} \\
(\mathrm{PT}+)\end{array}$ & $\mathrm{N}$ & Occupational exposure \\
\hline EM [115] & NR & NR & NR & $\mathrm{NR}$ & $\mathrm{N}$ & $\mathrm{N}$ & \\
\hline SJS [116] & 10 days & $62 \mathrm{y} / \mathrm{o} \mathrm{F}$ & $\mathrm{PO}$ & NR & $\mathrm{N}$ & $\mathrm{N}$ & \\
\hline $\mathrm{CD}[17]$ & 3 mo-4 yrs & $\begin{array}{l}>18 \mathrm{y} / \mathrm{o} \\
\text { gender NR }\end{array}$ & TOP & NR & $\begin{array}{c}\mathrm{Y} \\
(\mathrm{n}=4, \mathrm{PT}+)\end{array}$ & $\mathrm{N}$ & Occupational Exposure \\
\hline LABD [117] & 3 days & $54 \mathrm{y} / \mathrm{o} \mathrm{M}$ & $\mathrm{PO}$ & $\mathrm{N}$ & $\mathrm{N}$ & $\mathrm{N}$ & \\
\hline $\begin{array}{c}\text { MPR } \\
\text { DRESS } \\
\text { Myocarditis [118] }\end{array}$ & $\begin{array}{c}\mathrm{H} \\
7 \text { days } \\
2 \text { mo. }\end{array}$ & $48 \mathrm{y} / 0 \mathrm{M}$ & $\mathrm{PO}$ & NR & $\mathrm{N}$ & $\mathrm{N}$ & Skin and heart biopsy proven \\
\hline CD [18] & 28 days & $39 \mathrm{y} / \mathrm{o} \mathrm{M}$ & TOP & NR & $\begin{array}{c}\mathrm{Y} \\
(\mathrm{A}, \mathrm{PT}+\text { + } \mathrm{C}, \mathrm{PT}-)\end{array}$ & $\mathrm{N}$ & $\begin{array}{l}\text { No cross reactivity with } A \text { and } C \\
\text { Occupational Exposure }\end{array}$ \\
\hline CD [119] & 21 days & $76 \mathrm{y} / \mathrm{oF}$ & OPTH & $\begin{array}{c}\mathrm{Y} \\
\text { (tobramycin/dexamethasone) }\end{array}$ & $\begin{array}{c}\mathrm{Y} \\
(\mathrm{PT}+)\end{array}$ & $\mathrm{N}$ & No cross-reactivity with A and C \\
\hline
\end{tabular}


Table 3. Cont.

\begin{tabular}{|c|c|c|c|c|c|c|c|}
\hline Reaction & Time of Onset \# & Demographics & Dosage Form & Concomitant Agents & Allergy Evaluation/Confirmation & Prior Sensitization & Notes \\
\hline DRESS [120] & 5 days & $8 \mathrm{y} / \mathrm{o} \mathrm{M}$ & $\mathrm{PO}$ & $\mathrm{N}$ & $\mathrm{N}$ & $\mathrm{N}$ & \\
\hline Anaphylaxis [121] & 2 days & $\begin{array}{c}8 \mathrm{y} / \mathrm{o} \\
\text { gender NR }\end{array}$ & IV & NR & $\begin{array}{c}\mathrm{Y} \\
(\mathrm{SPT}+, \mathrm{IDT}+)\end{array}$ & NR & \\
\hline Anaphylaxis [121] & 2 days & $\begin{array}{c}9 \mathrm{y} / \mathrm{o} \\
\text { gender NR }\end{array}$ & PO & NR & $\begin{array}{c}\mathrm{Y} \\
(\mathrm{SPT}+, \mathrm{IDT}+) \\
\end{array}$ & $\begin{array}{c}\mathrm{Y} \\
(\mathrm{C}, 1 \mathrm{yr} \text { prior same reaction }) \\
\end{array}$ & Cross reactivity with $A$ and $C$. \\
\hline Anaphylaxis [121] & 1 day & $\begin{array}{c}7 \mathrm{y} / \mathrm{o} \\
\text { gender NR }\end{array}$ & $\mathrm{PO}$ & NR & $\begin{array}{c}\mathrm{Y} \\
(\mathrm{SPT}-, \mathrm{IDT}+)\end{array}$ & Unknown & \\
\hline MPE [122] & 2 days & $23 \mathrm{y} / \mathrm{o} \mathrm{M}$ & $\mathrm{PO}$ & $\mathrm{N}$ & $\mathrm{N}$ & $\mathrm{N}$ & Coinfected mononucleosis \\
\hline CD [123] & NR & $85 \mathrm{y} / \mathrm{oF}$ & OPTH & $\mathrm{N}$ & $\begin{array}{c}\mathrm{Y} \\
(\mathrm{A}, \mathrm{PT}+; \mathrm{C} \text { and } \mathrm{E} \text { PT- }) \\
\end{array}$ & $\begin{array}{c}\mathrm{Y} \\
\text { (A, 1 yr prior no reaction) }\end{array}$ & No cross reactivity with $\mathrm{A}, \mathrm{C}, \mathrm{E}$. \\
\hline DRESS [124] & 7 days & $44 \mathrm{y} / \mathrm{o} \mathrm{M}$ & PO & $\begin{array}{c}\mathrm{Y} \\
\text { (promethazine, dextromethorphan) }\end{array}$ & $\mathrm{N}$ & $\mathrm{N}$ & \\
\hline AGEP [125] & 2 days & $18 \mathrm{y} / \mathrm{o} \mathrm{F}$ & PO & $\mathrm{N}$ & $\mathrm{N}$ & $\mathrm{N}$ & \\
\hline FDE [126] & $<24 \mathrm{~h}$ & $35 \mathrm{y} / \mathrm{o} \mathrm{M}$ & PO & $\mathrm{N}$ & $\begin{array}{c}\mathrm{Y} \\
(\mathrm{DPT}+) \\
\end{array}$ & $\begin{array}{c}\mathrm{Y} \\
\text { (A, same reaction } \sim 12 \text { mo. apart) }\end{array}$ & \\
\hline SJS [127] & 1 day & $58 \mathrm{y} / \mathrm{o} \mathrm{M}$ & $\mathrm{PO}$ & $\begin{array}{c}\mathrm{Y} \\
\begin{array}{c}\text { (atenolol, atorvastatin, famotidine, } \\
\text { prednisone, hydroxyzine) }\end{array} \\
\end{array}$ & $\mathrm{N}$ & $\mathrm{N}$ & \\
\hline AGEP [128] & 1 day & $71 \mathrm{y} / \mathrm{o} \mathrm{F}$ & $\mathrm{PO}$ & NR & $\begin{array}{c}\mathrm{Y} \\
\text { (A, PT-, DPT+; E PT-; } \\
\text { C PT- and C DPT-) } \\
\end{array}$ & NR & No cross reactivity with $\mathrm{A}, \mathrm{C}, \mathrm{E}$. \\
\hline DRESS [129] & 4 days & $1.4 \mathrm{yrs} \mathrm{M}$ & $\mathrm{PO}$ & $\begin{array}{c}\mathrm{Y} \\
\text { (pranlukast) }\end{array}$ & $\mathrm{N}$ & $\mathrm{N}$ & \\
\hline FDE [130] & 1 day & $50 \mathrm{y} / \mathrm{o} \mathrm{F}$ & $\mathrm{PO}$ & $\mathrm{N}$ & & $\begin{array}{c}\mathrm{Y} \\
\text { (A, same reaction } 2 \text { yrs prior) }\end{array}$ & \\
\hline SJS [131] & 1 day & $6 \mathrm{y} / \mathrm{o} \mathrm{M}$ & $\mathrm{PO}$ & $\begin{array}{c}\mathrm{Y} \\
\text { (cefmetazole) }\end{array}$ & $\mathrm{N}$ & $\mathrm{N}$ & \\
\hline
\end{tabular}

Abbreviations: $\mathrm{ACDR}=$ acute cutaneous drug reaction ${ }^{\mathrm{a}} ; \mathrm{ADR}=$ adverse drug reaction; $\mathrm{AGEP}=$ acute generalized exanthematous pustulosis; $\mathrm{APAP}=$ acetaminophen; $\mathrm{ASA}=$ aspirin; $\mathrm{A}=$ azithromycin; $\mathrm{C}=$ clarithromycin; $\mathrm{CD}=$ contact dermatitis; $\mathrm{DR}=$ delayed reaction; $\mathrm{DPT}=$ drug provocation test; $\mathrm{DRESS}=$ Drug reaction with eosinophilia and systemic symptoms syndrome; $\mathrm{E}=$ erythromycin; $\mathrm{EM}=$ erythema multiforme; $\mathrm{ER}=$ extended release; $\mathrm{ETH}=$ ethambutol; $\mathrm{F}=$ female; FDX = fidaxomicin; HRT = leukocyte histamine release test; HSP = Henoch-Schönlein Purpura; $\mathrm{h}=$ hour(s); HCTZ = hydrochlorothiazide; HIV = human immunodeficiency virus; IBU = ibuprofen; IDT = intradermal test; Ig E = immunoglobulin $\mathrm{E} ; \mathrm{IM}=$ intramuscular; $\mathrm{IR}=$ immediate reaction $(<1 \mathrm{~h}) ; \mathrm{LABD}=$ Linear Immunoglobulin A Bullous Dermatosis; $\mathrm{LCV}=$ leukocytoclastic vasculitis; $\mathrm{LTT}=$ lymphocyte transformation test; $\mathrm{MAS}=$ multiple allergy sensitivity; $\mathrm{MDT}=$ mast cell degranulation test; min= minutes; $\mathrm{mo}=$ months; MMIT = macrophage migration inhibitory test; MPE $=$ maculopapular exanthema; $\mathrm{MPR}=$ maculopapular rash; $\mathrm{N}=$ no; $\mathrm{NIR}=$ nonimmediate reaction $(1-72 \mathrm{~h}) ; \mathrm{NR}=$ not reported; $\mathrm{NTG}=$ nitroglycerin; opth $=$ ophthalmic; $\mathrm{PT}=$ patch test; $\mathrm{R}=$ roxithromycin; $\mathrm{RIF}=$ rifampin; RFB = rifabutin; $\mathrm{S}=$ spiramycin; SJS = Steven's Johnson syndrome; SOB = shortness of breath; SPT = skin prick test; ST = scratch test; TEN = toxic epidermal necrolysis; $\mathrm{TMP} / \mathrm{SMX}=$ trimethoprim/sulfamethoxazole; top = topical; $\mathrm{wks}=$ weeks; $\mathrm{Y}=$ yes; $\mathrm{yrs}=$ years; $\mathrm{y} / \mathrm{o}=$ years old. * Exact time course unknown as awoke with FDE. \# Time of onset means time when symptoms began to occur either during macrolide therapy or after completion of macrolide therapy'ACDR defined as allergic reaction, adverse drug reaction, pruritis, general swelling, local or general redness, erythema, rash, urticaria, or other skin disease. ${ }^{\pi}$ Review was meant to include azithromycin, clarithromycin, erythromycin, and fidaxomicin; however, there is a chance these data include excluded macrolides since the type of macrolides reported were not fully specified 
Table 4. Summary of published literature reporting fidaxomycin hypersensitivity.

\begin{tabular}{|c|c|c|c|c|c|c|c|}
\hline Reaction & Time of Onset ${ }^{\#}$ & Demographics & Dosage Form & Concomitant Agents & Allergy Evaluation/Confirmation & Prior Sensitization & Notes \\
\hline Diffuse rash [132] & 5 days & 73 y/o M & $\mathrm{PO}$ & NR & $\mathrm{N}$ & $\mathrm{N}$ & \\
\hline $\begin{array}{l}\text { Rash, swollen } \\
\text { eyes and lips [132] }\end{array}$ & $1-2 \mathrm{~h}$ & $74 \mathrm{y} / \mathrm{oF}$ & $\mathrm{PO}$ & NR & $\mathrm{N}$ & $\mathrm{N}$ & \\
\hline Diffuse rash [132] & 5 days & $26 \mathrm{y} / \mathrm{o} \mathrm{F}$ & PO & NR & $\mathrm{N}$ & $\mathrm{N}$ & \\
\hline Diffuse rash and itching [132] & $1 \mathrm{~h}$ & $49 \mathrm{y} / \mathrm{o} \mathrm{F}$ & $\mathrm{PO}$ & NR & $\mathrm{N}$ & $\begin{array}{c}\mathrm{Y} \\
\text { (E and A allergy) }\end{array}$ & $\begin{array}{l}\text { Cross reactivity } \\
\text { with FDX, E, A. }\end{array}$ \\
\hline Throat burning [132] & $12-24 \mathrm{~h}$ & $79 \mathrm{y} / \mathrm{o} \mathrm{F}$ & $\mathrm{PO}$ & NR & $\mathrm{N}$ & $\begin{array}{c}\mathrm{Y} \\
\text { (E allergy) }\end{array}$ & \\
\hline Throat and chest swelling [132] & 2 days; $N$ & F, age NR & $\mathrm{PO}$ & NR & $\begin{array}{c}\mathrm{Y} \\
(\mathrm{DPT}+)\end{array}$ & $\begin{array}{c}\mathrm{Y} \\
\text { (F } 30 \text { days prior; E allergy) }\end{array}$ & \\
\hline $\begin{array}{l}\text { Eyes, lips, mouth swelling, } \\
\text { itchy face [132] }\end{array}$ & $72 \mathrm{~h}$ & $53 \mathrm{y} / \mathrm{oF}$ & $\mathrm{PO}$ & NR & $\mathrm{N}$ & $\mathrm{N}$ & \\
\hline Angioedema [132] & $\begin{array}{l}5 \text { days, } \\
6 \text { days }\end{array}$ & $56 \mathrm{y} / \mathrm{o} \mathrm{M}$ & $\mathrm{PO}$ & NR & $\begin{array}{c}\mathrm{Y} \\
(\mathrm{DPT}+)\end{array}$ & $\begin{array}{c}\mathrm{Y} \\
\text { (FDX same reaction within } 24 \mathrm{~h} \text { ) }\end{array}$ & \\
\hline Lip swelling [132] & 7 days & F, age NR & PO & NR & $\mathrm{N}$ & $\mathrm{N}$ & \\
\hline Severe rash [132] & NR & M, age NR & $\mathrm{PO}$ & NR & $\mathrm{N}$ & $\mathrm{N}$ & \\
\hline $\begin{array}{l}\text { SOB, throat swelling, } \\
\text { chest tightness [132] }\end{array}$ & 2 days & $50 \mathrm{y} / \mathrm{oF}$ & $\mathrm{PO}$ & NR & $\mathrm{N}$ & $\mathrm{N}$ & \\
\hline BLE edema and rash [132] & 5 days & $70 \mathrm{y} / \mathrm{o} \mathrm{M}$ & $\mathrm{PO}$ & NR & $\mathrm{N}$ & $\mathrm{N}$ & \\
\hline MAS [46] & NR & $\begin{array}{c}\mathrm{n}=6,<18 \mathrm{y} / \mathrm{o} \text { and } \\
\text { gender NR }\end{array}$ & $\mathrm{PO}$ & $\mathrm{N}$ & $\begin{array}{c}\mathrm{Y} \\
(\mathrm{PT}+, \mathrm{DPT}+)\end{array}$ & NR & \\
\hline ACDR [77] & NR & NR & NR & NR & $\mathrm{N}$ & NR & \\
\hline MAS [133] & NR & $\begin{array}{l}\mathrm{n}=34, \text { age and } \\
\text { gender NR }\end{array}$ & NR & NR & $\mathrm{N}$ & NR & \\
\hline + DPT with ADR [134] & NR & $\begin{array}{c}\mathrm{n}=102, \text { age and } \\
\text { gender NR }\end{array}$ & NR & NR & $\begin{array}{c}\mathrm{Y} \\
(\mathrm{n}=14, \mathrm{DPT}+)\end{array}$ & NR & \\
\hline ADRs [97] & NR & $\begin{array}{c}\mathrm{n}=4(<18 \mathrm{y} / \mathrm{o}), \\
\text { gender NR }\end{array}$ & NR & NR & $\begin{array}{c}\mathrm{Y} \\
(\mathrm{NR})\end{array}$ & NR & \\
\hline
\end{tabular}

Abbreviations: $\mathrm{ACDR}=$ acute cutaneous drug reaction ${ }^{\mathrm{a}}$; $\mathrm{ADR}=$ adverse drug reaction; $\mathrm{AGEP}=$ acute generalized exanthematous pustulosis; $\mathrm{APAP}=$ acetaminophen; $\mathrm{ASA}=$ aspirin; $\mathrm{A}=$ azithromycin; $\mathrm{C}=$ clarithromycin; $\mathrm{CD}=$ contact dermatitis; $\mathrm{DR}=$ delayed reaction; $\mathrm{DPT}=$ drug provocation test; DRESS = Drug reaction with eosinophilia and systemic symptoms $\mathrm{A}=$ azithromycin; $\mathrm{C}=$ clarithromycin; $\mathrm{CD}=$ contact dermatitis; $\mathrm{DR}=$ delayed reaction; $\mathrm{DPT}=$ drug provocation test; $\mathrm{DRESS}=\mathrm{Drug}$ reaction with eosinophilia and systemic symptoms syndrome; $\mathrm{E}=$ erythromycin; $\mathrm{EM}=$ erythema multiforme; $\mathrm{ER}=$ extended release; $\mathrm{ETH}=$ ethambutol; $\mathrm{F}=$ female; FDX = fidaxomicin; HRT = leukocyte histamine release test;
$\mathrm{HSP}=$ Henoch-Schönlein Purpura; $\mathrm{h}=$ hour(s); HCTZ = hydrochlorothiazide; HIV = human immunodeficiency virus; IBU = ibuprofen; IDT = intradermal test; Ig E = immunoglobulin E; IM = intramuscular; IR = immediate reaction $(<1 \mathrm{~h})$; LABD = Linear Immunoglobulin A Bullous Dermatosis; LCV = leukocytoclastic vasculitis; LTT = lymphocyte transformation test; MAS = multiple allergy sensitivity; MDT = mast cell degranulation test; min= minutes; $\mathrm{mo}=$ months; MMIT = macrophage migration inhibitory test; MPE $=$ maculopapular exanthema; $\mathrm{MPR}=$ maculopapular rash; $\mathrm{N}=$ no; NIR= nonimmediate reaction $(1-72 \mathrm{~h}) ; \mathrm{NR}=$ not reported; $\mathrm{NTG}=$ nitroglycerin; opth = ophthalmic; $\mathrm{PT}=$ patch test; $\mathrm{R}=$ roxithromycin; $\mathrm{RIF}=$ rifampin; RFB = rifabutin; $\mathrm{S}=$ spiramycin; $\mathrm{SJS}=$ Steven's Johnson syndrome; $\mathrm{SOB}=$ shortness of breath; $\mathrm{SPT}=$ skin prick test; $\mathrm{ST}=\mathrm{scratch}$ test; $\mathrm{TEN}=$ toxic epidermal necrolysis; $\mathrm{TMP} / \mathrm{SMX}=$ trimethoprim/sulfamethoxazole; top = topical; $\mathrm{wks}=$ weeks; $\mathrm{Y}=$ yes; yrs = years; $\mathrm{y} / \mathrm{o}=$ years old. * Exact time course unknown as awoke with FDE. \# Time of onset means time when symptoms began to occur either during macrolide therapy or after completion of macrolide therapy. ACDR defined as allergic reaction, adverse drug reaction, pruritis, general swelling, local or general redness, erythema, rash, urticaria, or other skin disease. ${ }^{\pi}$ Review was meant to include azithromycin, clarithromycin, erythromycin, and fidaxomicin; however, there is a chance these data include excluded macrolides since the type of macrolides reported were not fully specified. 
Macrolide allergies are rare and available desensitization protocols are restricted to case reports, which all demonstrated success $[74,100,102]$. Three cases involved successful desensitization with clarithromycin, and one of the patients also completed a desensitization for azithromycin desensitization. Four desensitization protocols were identified in the literature: two involving clarithromycin in an adult patient, one for clarithromycin in a pediatric patient, and one for azithromycin in a pediatric patient (Appendix A, Tables A1-A4) [74,100,102]. Holmes et al. reported a case of a 68-year-old female with a history of anaphylaxis with erythromycin and bronchospasms with roxithromycin who underwent oral clarithromycin desensitization for use in a 18 month treatment course for a skin infection caused by Mycobacterium chelonae (Appendix A, Table A1) [74]. Swamy et al. reported a case of a 68-year-old female with a history of anaphylaxis with azithromycin and urticaria and bronchospasms with clarithromycin who underwent oral clarithromycin desensitization for use in a 3 month treatment course for an infection caused by Mycobacterium intracellularae (Appendix A, Table A2) [100]. Repeated intradermal skin testing with azithromycin $1 \mathrm{~h}$ after desensitization demonstrated a similar reaction seen at baseline. Six weeks after desensitization, azithromycin and clarithromycin skin prick tests were negative and intradermal skin tests were equivocal to the reactions seen at baseline. Petitto et al. described a case of an 11-year-old female with a history of diffuse urticaria with clarithromycin who underwent successful macrolide desensitization for use in osteomyelitis caused by Mycobacterium fortuitum (Appendix A, Tables A3 and A4) [102]. Initially, this patient completed an azithromycin desensitization protocol without complication; however, $24 \mathrm{~h}$ later, she developed a generalized urticarial rash within $75 \mathrm{~min}$ of a treatment dose. One week later, this patient underwent oral clarithromycin desensitization and all subsequent treatment doses were tolerated.

\section{Conclusions}

Macrolides are infrequently reported to cause various types of allergic reactions, with cutaneous reactions being the most common. Macrolides are similar in chemical structure, and limited reports have demonstrated cross-reactivity. Strategies to overcome hypersensitivity reactions, such as desensitization, or allergy testing for cross-reactivity to another macrolide have been utilized with successful outcomes. Consequently, if a patient has a severe hypersensitivity reaction to a macrolide, then the benefit versus the risk must be evaluated for using an agent in this class. In clinical practice, it may be more convenient and safer to change to an alternative class of medications if the option is available. However, if a macrolide must be used in a patient with a confirmed history of a severe allergic reaction, then allergy testing should be employed to investigate if the patient reacts to other agents in the macrolide class. If so, a desensitization may be conducted while the patient is monitored closely for signs and symptoms of an allergic reaction.

Author Contributions: Conceptualization, K.M.S., J.C., E.B.C.; methodology, K.M.S., J.C.C., E.B.C.; writingoriginal draft preparation, K.M.S., J.C.C., E.B.C., and S.V.G.; writing-review and editing, K.M.S., J.C.C., E.B.C., and S.V.G.

Funding: This research received no external funding.

Conflicts of Interest: KMS and SVG declare no conflicts of interest. EBC serves on the speakers' bureau for Merck $\& \mathrm{Co}$, Inc. JCCCho serves on the speakers' bureau for Allergan, Inc. 


\section{Appendix A}

Table A1. Oral clarithromycin desensitization protocol in an adult [74] *.

\begin{tabular}{cccc}
\hline \multirow{2}{*}{ Dose No. } & $\begin{array}{c}\text { Concentration } \\
(\mathbf{m g} / \mathbf{m L})\end{array}$ & $\mathbf{3}$ & $\mathbf{m g}$ \\
\cline { 3 - 4 } & & 0.1 & 0.005 \\
1. & 0.05 & 0.2 & 0.010 \\
2. & 0.05 & 0.4 & 0.020 \\
3. & 0.05 & 1 & 0.050 \\
4. & 0.05 & 2 & 0.100 \\
5. & 0.05 & 4 & 0.200 \\
6. & 0.05 & 0.8 & 0.400 \\
7. & 0.50 & 1.6 & 0.800 \\
8. & 0.50 & 3.2 & 1.6 \\
9. & 0.50 & 6.4 & 3.2 \\
10. & 0.50 & 1.2 & 6 \\
11. & 5 & 2.4 & 12 \\
12. & 5 & 4.8 & 24 \\
13. & 5 & 1 & 50 \\
14. & 50 & 2 & 100 \\
15. & 50 & 4 & 200 \\
16. & 50 & 8 & 400 \\
17. & 50 & 10 & 500 \\
18. & 50 & & 1298.4 \\
\hline
\end{tabular}

Abbreviations: $\mathrm{mg}=$ milligrams; $\mathrm{mL}=$ milliliters; $\mathrm{no}=$ number. ${ }^{*}$ Serial 10 -fold dilutions of a clarithromycin suspension of $125 \mathrm{mg} / 5 \mathrm{~mL}(25 \mathrm{mg} / \mathrm{mL})$ were performed to make clarithromycin solutions at $2.5,0.25$, and $0.025 \mathrm{mg} / \mathrm{mL}$. Each dose was administered in 15-min intervals over $4.5 \mathrm{~h}$ with close monitoring on the intensive care unit (for $36 \mathrm{~h}$ ).

Table A2. Oral clarithromycin desensitization protocol in an adult [100] *.

\begin{tabular}{cccc}
\hline \multirow{2}{*}{ Dose No. } & $\begin{array}{c}\text { Concentration } \\
(\mathbf{m g} / \mathbf{m L})\end{array}$ & $\mathbf{m L}$ & $\mathbf{m g}$ \\
\cline { 3 - 4 } & & 1.25 & 0.030 \\
1. & 0.025 & 2.50 & 0.060 \\
2. & 0.025 & 5 & 0.125 \\
3. & 0.025 & 1 & 0.250 \\
4. & 0.250 & 2 & 0.5 \\
5. & 0.250 & 4 & 1 \\
6. & 0.250 & 0.8 & 2 \\
7. & 2.5 & 1.6 & 4 \\
8. & 2.5 & 3.2 & 8 \\
9. & 2.5 & 6.4 & 16 \\
10. & 2.5 & 1.3 & 32 \\
11. & 25 & 2.5 & 64 \\
12. & 25 & 5 & 125 \\
13. & 25 & 10 & 250 \\
14. & 25 & & 503 \\
\hline
\end{tabular}

Abbreviations: $\mathrm{mg}=$ milligrams; $\mathrm{mL}=$ milliliters; no = number. * Serial 10-fold dilutions of a clarithromycin suspension of $125 \mathrm{mg} / 5 \mathrm{~mL}(25 \mathrm{mg} / \mathrm{mL})$ were performed to make clarithromycin solutions at $2.5,0.25$, and $0.025 \mathrm{mg} / \mathrm{mL}$. Each dose was administered in 15-min intervals over $3.5 \mathrm{~h}$. 
Table A3. Azithromycin oral desensitization protocol in an adolescent [102].

\begin{tabular}{cccc}
\hline \multirow{2}{*}{ Dose No. } & $\begin{array}{c}\text { Concentration } \\
(\mathbf{m g} / \mathbf{m L})\end{array}$ & $\mathbf{m L}$ & $\mathbf{m g}$ \\
\cline { 3 - 4 } & & 0.6 & 0.030 \\
1. & 0.025 & 1.2 & 0.060 \\
2. & 0.025 & 2.5 & 0.125 \\
3. & 0.025 & 5 & 0.250 \\
4. & 0.250 & 1 & 0.500 \\
5. & 0.250 & 2 & 1 \\
6. & 0.250 & 4 & 2 \\
7. & 2.5 & 0.8 & 4 \\
8. & 2.5 & 1.6 & 8 \\
9. & 2.5 & 3.2 & 16 \\
10. & 2.5 & 6.4 & 32 \\
11. & 25 & 2.5 & 64 \\
12. & 25 & 2.5 & 125 \\
13. & 25 & & 253 \\
\hline
\end{tabular}

Table A4. Clarithromycin oral desensitization protocol in an adolescent [102].

\begin{tabular}{cccc}
\hline \multirow{2}{*}{ Dose No. } & $\begin{array}{c}\text { Concentration } \\
(\mathbf{m g} / \mathbf{m L})\end{array}$ & $\mathbf{m L}$ & $\mathbf{m g}$ \\
\cline { 3 - 4 } & & 0.60 & 0.030 \\
1. & 0.05 & 1.20 & 0.060 \\
2. & 0.05 & 2.50 & 0.125 \\
3. & 0.05 & 5 & 0.25 \\
4. & 0.05 & 1 & 0.50 \\
5. & 0.50 & 2 & 1 \\
6. & 0.50 & 4 & 2 \\
7. & 0.50 & 0.8 & 4 \\
8. & 5 & 1.6 & 8 \\
9. & 5 & 3.2 & 16 \\
10. & 5 & 6.4 & 32 \\
11. & 5 & 2.5 & 125 \\
12. & 50 & 2.5 & 125 \\
13. & 50 & & 314 \\
\hline
\end{tabular}

\section{References}

1. Blumenthal, K.G.; Peter, J.G.; Trubiano, J.A.; Phillips, E.J. Antibiotic allergy. Lancet 2019, 393, 183-198. [CrossRef]

2. Sanchez-Borges, M.; Thong, B.; Blanca, M.; Ensina, L.F.; Gonzalez-Diaz, S.; Greenberger, P.A.; Jares, E.; Jee, Y.K.; Kase-Tanno, L.; Khan, D.; et al. Hypersensitivity reactions to non beta-lactam antimicrobial agents, a statement of the WAO special committee on drug allergy. World Allergy Organ J. 2013, 6, 18. [CrossRef] [PubMed]

3. Thong, B.Y. Update on the management of antibiotic allergy. Allergy Asthma Immunol Res. 2010, 2, 77-86. [CrossRef] [PubMed]

4. Araujo, L.; Demoly, P. Macrolides allergy. Curr. Pharm. Des. 2008, 14, 2840-2862. [CrossRef] [PubMed]

5. Dinos, G.P. The macrolide antibiotic renaissance. Br. J. Pharmacol. 2017, 174, 2967-2983. [CrossRef] [PubMed]

6. Brisson-Noel, A.; Trieu-Cuot, P.; Courvalin, P. Mechanism of action of spiramycin and other macrolides. J. Antimicrob. Chemother. 1988, 22 (Suppl. B), 13-23. [CrossRef]

7. Zhanel, G.G.; Walkty, A.J.; Karlowsky, J.A. Fidaxomicin: A novel agent for the treatment of Clostridium difficile infection. Can. J. Infect. Dis. Med. Microbiol. 2015, 26, 305-312. [CrossRef] [PubMed]

8. Romano, A.; Caubet, J.C. Antibiotic allergies in children and adults: From clinical symptoms to skin testing diagnosis. J. Allergy Clin. Immunol. Pract. 2014, 2, 3-12. [CrossRef] 
9. Sivapalasingam, S.; Steigbigel, N.H. Macrolides. In Mandell, Douglas, and Bennett's Principles and Practice of Infectious Diseases, 7th ed.; Mandell, G.L., Bennett, J.E., Dolin, R., Eds.; Macrolides, Clindamycin, and Ketolides; Churchill Livingstone/Elsevier: Philadelphia, PA, USA, 2010; Available online: http://web.a.ebscohost. com.ezproxy.lib.usf.edu/ehost/detail/detail?vid=0\&sid=ab19e82b-d5a8-4468-ab6a-243ed20974fa\%40sdcv-sessmgr04\&bdata=JnNpdGU9ZWhvc3QtbGl2ZQ\%3d\%3d\#AN=458761\&db=nlebk （accessed on 29 May 2019).

10. Bradley, J.S.; Byington, C.L.; Shah, S.S.; Alverson, B.; Carter, E.R.; Harrison, C.; Kaplan, S.L.; Mace, S.E.; McCracken, G.H., Jr.; Moore, M.R.; et al. Pediatric Infectious Diseases, S. the Infectious Diseases Society of, A. Executive summary: The management of community-acquired pneumonia in infants and children older than 3 months of age: Clinical practice guidelines by the Pediatric Infectious Diseases Society and the Infectious Diseases Society of America. Clin. Infect. Dis. 2011, 53, 617-630.

11. Mandell, L.A.; Wunderink, R.G.; Anzueto, A.; Bartlett, J.G.; Campbell, G.D.; Dean, N.C.; Dowell, S.F.; File, T.M., Jr.; Musher, D.M.; Niederman, M.S.; et al. Infectious Diseases Society of America/American Thoracic Society consensus guidelines on the management of community-acquired pneumonia in adults. Clin. Infect. Dis. 2007, 44 (Suppl. S2), 27-72. [CrossRef]

12. Lieberthal, A.S.; Carroll, A.E.; Chonmaitree, T.; Ganiats, T.G.; Hoberman, A.; Jackson, M.A.; Joffe, M.D.; Miller, D.T.; Rosenfeld, R.M.; Sevilla, X.D.; et al. The diagnosis and management of acute otitis media. Pediatrics 2013, 131, e964-e999. [CrossRef] [PubMed]

13. Shulman, S.T.; Bisno, A.L.; Clegg, H.W.; Gerber, M.A.; Kaplan, E.L.; Lee, G.; Martin, J.M.; Van Beneden, C. Clinical practice guideline for the diagnosis and management of group A streptococcal pharyngitis: 2012 update by the Infectious Diseases Society of America. Clin. Infect. Dis. 2012, 55, 1279-1282. [CrossRef] [PubMed]

14. Chahine, E.B.; Sucher, A.J.; Mantei, K. Fidaxomicin: A novel macrolide antibiotic for Clostridium difficile infection. Consult. Pharm. 2014, 29, 614-624. [CrossRef] [PubMed]

15. McDonald, L.C.; Gerding, D.N.; Johnson, S.; Bakken, J.S.; Carroll, K.C.; Coffin, S.E.; Dubberke, E.R.; Garey, K.W.; Gould, C.V.; Kelly, C.; et al. Clinical Practice Guidelines for Clostridium difficile Infection in Adults and Children: 2017 Update by the Infectious Diseases Society of America (IDSA) and Society for Healthcare Epidemiology of America (SHEA). Clin. Infect. Dis. 2018, 66, 987-994. [CrossRef] [PubMed]

16. Mimesh, S.; Pratt, M. Occupational airborne allergic contact dermatitis from azithromycin. Contact Dermat. 2004, 51, 151. [CrossRef] [PubMed]

17. Milkovic-Kraus, S.; Macan, J.; Kanceljak-Macan, B. Occupational allergic contact dermatitis from azithromycin in pharmaceutical workers: A case series. Contact Dermat. 2007, 56, 99-102. [CrossRef] [PubMed]

18. Lopez-Lerma, I.; Romaguera, C.; Vilaplana, J. Occupational airborne contact dermatitis from azithromycin. Clin. Exp. Derm. 2009, 34, e358-e359. [CrossRef] [PubMed]

19. Bower, A.G. Anaphylactic death from erythromycin. Calif. Med. 1958, 89, 279-280.

20. Prasad, A.S. Severe urticaria following erythromycin therapy. Acta Med. Iran 1960, 3, 9-11. [CrossRef]

21. Crawford, L.V.; Roane, J. Use of erythromycin ethyl succinate in allergic children. Ann. Allergy 1969, $27,18-22$.

22. Grainger, G.J. Erythromycin estolate in general practice. A comparative trial of two dosage forms. Practitioner 1969, 202, 84-86. [PubMed]

23. Abramov, L.A.; Yust, I.C.; Fierstater, E.M.; Vardinon, N.E. Acute Respiratory Distress Caused by Erythromycin Hypersensitivity. Arch. Intern. Med. 1972, 138, 1156-1158. [CrossRef]

24. Handa, S.P. The Schonlein-Henoch syndrome: Glomerulonephritis following erythromycin. South Med. J 1972, 65, 917-920. [CrossRef] [PubMed]

25. Naik, R.P.; Singh, G. Bullous fixed drug eruption presumably due to erythromycin. Dermatologica 1976, 152, 177-180. [CrossRef] [PubMed]

26. Van Ketel, W.G. Immediate-and delayed-type allergy to erythromycin. Contact Dermat. 1976, 2, 363-364. [CrossRef] [PubMed]

27. Cooksley, W.G.; Powell, L.W. Erythromycin jaundice: Diagnosis by an in vitro challenge test. Aust. N. Z. J. Med. 1977, 7, 291-293. [CrossRef]

28. Lloyd-Still, J.D.; Sherman, J.O.; Boggs, J.; Demers, L.M. Erythromycin estolate hepatotoxicity. Am J Dis. Child 1978, 132, 320. [CrossRef]

29. Kirby, B.D.; Snyder, K.M.; Meyer, R.D.; Finegold, S.M. Legionnaires' disease: Clinical features of 24 cases. Ann. Intern Med. 1978, 89, 297-309. [CrossRef]

30. Harris, R.J.; Harris, R.L. Multiple antibiotic allergies. J. Am. Dent. Assoc. 1978, 97, 994-995. [CrossRef] 
31. Zafrani, E.S.; Ishak, K.G.; Rudzki, C. Cholestatic and hepatocellular injury associated with erythromycin esters: Report of nine cases. Dig Dis. Sci. 1979, 24, 385-396. [CrossRef]

32. Hartigan, D.A.; Toma, S.; Listgarten, C. Legionnaires' disease caused by Legionella pneumophila serogroup 3. Lancet 1979, 2, 411. [CrossRef]

33. Schonheyder, H. Stevens-Johnson syndrome associated with intrahepatic cholestasis and respiratory disease: A case report. Acta Derm. Venereol. 1981, 61, 171-173. [PubMed]

34. Keeffe, E.B.; Reis, T.C.; Berland, J.E. Hepatotoxicity to both erythromycin estolate and erythromycin ethylsuccinate. Dig. Dis. Sci. 1982, 27, 701-704. [CrossRef] [PubMed]

35. Lombardi, P.; Campolmo, P.; Spallanzani, P.; Sertoli, A. Delayed hypersensitivity to erythromycin. Contact Dermat. 1982, 8, 416. [CrossRef] [PubMed]

36. Rosenfeld, J.; Gura, V.; Boner, G.; Ben-Bassat, M.; Livni, E. Interstitial nephritis with acute renal failure after erythromycin. Br. Med. J. (Clin. Res. Ed.) 1983, 286, 938-939. [CrossRef] [PubMed]

37. Furniss, L.D. Rash due to erythromycin. Drug Intell. Clin. Pharm. 1983, 17, 631.

38. Diehl, A.M.; Latham, P.; Boitnott, J.K.; Mann, J.; Maddrey, W.C. Cholestatic hepatitis from erythromycin ethylsuccinate. Report of two cases. Am. J. Med. 1984, 76, 931-934. [CrossRef]

39. Pigatto, P.D.; Riboldi, A.; Riva, F.; Altomare, G.F. Fixed drug eruption to erythromycin. Acta Derm. Venereol. 1984, 64, 272-273.

40. Lund Kofoed, M.; Oxholm, A. Toxic epidermal necrolysis due to erythromycin. Contact Dermat. 1985, 13, 273. [CrossRef]

41. Singer, D.R.; Simpson, J.G.; Catto, G.R.; Johnston, A.W. Drug hypersensitivity causing granulomatous interstitial nephritis. Am. J. Kidney Dis. 1988, 11, 357-359. [CrossRef]

42. Rigauts, H.D.; Selleslag, D.L.; Van Eyken, P.L.; Van Damme, B.J.; Fevery, J.M.; Marchal, G.J. Erythromycininduced hepatitis: Simulator of malignancy. Radiology 1988, 169, 661-662. [CrossRef] [PubMed]

43. Pendleton, N.; Mallik, L.J.; Williams, J.G. Erythromycin rash in glandular fever. Br. J. Clin. Pract. 1989, 43, 464-465. [PubMed]

44. J.acqz-Aigrain, E.; Guillonneau, M.; Brun, P.; Broyard, A.; Bechtel, Y. Toxic epidermal necrolysis due to paediatric erythromycin/sulfisoxazole combination. Lancet 1990, 336, 1010-1011. [CrossRef]

45. Florido Lopez, J.F.; Lopez Serrano, M.C.; Belchi Hernandez, J.; Estrada Rodriguez, J.L. Fixed eruption due to erythromycin. A case report. Allergy 1991, 46, 77-78. [CrossRef] [PubMed]

46. Kamada, M.M.; Twarog, F.; Leung, D.Y. Multiple antibiotic sensitivity in a pediatric population. Allergy Proc. 1991, 12, 347-350. [CrossRef] [PubMed]

47. Igea, J.M.; Quirce, S.; de la Hoz, B.; Fraj, J.; Pola, J.; Diez Gomez, M.L. Adverse cutaneous reactions due to macrolides. Ann. Allergy 1991, 66, 216-218. [PubMed]

48. Mutalik, S. Fixed drug eruption caused by erythromycin. Int. J. Derm. 1991, 30, 751. [CrossRef]

49. Shirin, H.; Schapiro, J.M.; Arber, N.; Pinkhas, J.; Sidi, Y.; Salomon, F. Erythromycin base-induced rash and liver function disturbances. Ann. Pharm. 1992, 26, 1522-1523. [CrossRef]

50. Lopez Serrano, C.; Quiralte Enriquez, J.; Martinez Alzamora, F. Urticaria from erythromycin. Allergol. Immunopathol. 1993, 21, 225-226.

51. Fernandez Redondo, V.; Casas, L.; Taboada, M.; Toribio, J. Systemic contact dermatitis from erythromycin. Contact Dermat. 1994, 30, 43-44. [CrossRef]

52. Pandha, H.S.; Dunn, P.J. Stevens-Johnson syndrome associated with erythromycin therapy. N. Z. Med. J. 1995, 108, 13. [PubMed]

53. Pascual, C.; Crespo, J.F.; Quiralte, J.; Lopez, C.; Wheeler, G.; Martin-Esteban, M. In vitro detection of specific IgE antibodies to erythromycin. J. Allergy Clin. Immunol. 1995, 95, 668-671. [CrossRef]

54. Moreau, A.; Dompmartin, A.; Castel, B.; Remond, B.; Leroy, D. Drug-induced acute generalized exanthematous pustulosis with positive patch tests. Int. J. Derm. 1995, 34, 263-266. [CrossRef] [PubMed]

55. Lestico, M.R.; Smith, A.D. Stevens-Johnson syndrome following erythromycin administration. Am. J. Health Syst. Pharm. 1995, 52, 1805-1807. [CrossRef] [PubMed]

56. Martins, C.; Freitas, J.D.; Goncalo, M.; Goncalo, S. Allergic contact dermatitis from erythromycin. Contact Dermat. 1995, 33, 360. [CrossRef] [PubMed]

57. Gomez-Lechon, M.J.; Carrasquer, J.; Berenguer, J.; Castell, J.V. Evidence of antibodies to erythromycin in serum of a patient following an episode of acute drug-induced hepatitis. Clin. Exp. Allergy 1996, 26, 590-596. [CrossRef] [PubMed] 
58. Valsecchi, R.; Pansera, B.; Reseghetti, A. Contact allergy to erythromycin. Contact Dermat. 1996, $34,428$. [CrossRef]

59. J.orro, G.; Morales, C.; Braso, J.V.; Pelaez, A. Anaphylaxis to erythromycin. Ann. Allergy Asthma Immunol. 1996, 77, 456-458. [CrossRef]

60. Goossens, C.; Sass, U.; Song, M. Baboon syndrome. Dermatology 1997, 194, 421-422. [CrossRef]

61. Alvarez-Ferrandez, J.A.; Alvarez-Cuesta, E.; Aragoneses, E.; Cuevas, M. Hypersensitivity to erythromycin: Cross Reactivity (A571). J. Allergy Clin. Immunol. 1998, 101, S138.

62. Quinones, M.D.; Sanchez, I.; Lopez, R.; Rodriguez, F.; Martin-Gil, D.; Santander, J.J. Pustulosis by Erythromycin. Allergy 1998, 53, 104.

63. Barbaud, A.; Reichert-Penetrat, S.; Trechot, P.; Jacquin-Petit, M.A.; Ehlinger, A.; Noirez, V.; Faure, G.C.; Schmutz, J.L.; Bene, M.C. The use of skin testing in the investigation of cutaneous adverse drug reactions. $\mathrm{Br}$. J. Derm. 1998, 139, 49-58. [CrossRef] [PubMed]

64. Mahboob, A.; Haroon, T.S. Drugs causing fixed eruptions: A study of 450 cases. Int. J. Derm. 1998, 37, $833-838$. [CrossRef] [PubMed]

65. Horn, S.; Aglas, F.; Horina, J.H. Cholestasis and liver cell damage due to hypersensitivity to erythromycin stearate-recurrence following therapy with erythromycin succinate. Wien. Klin. Wochenschr. 1999, 111, 76-77. [PubMed]

66. Gallardo, M.A.; Thomas, I. Hypersensitivity reaction to erythromycin. Cutis 1999, 64, 375-376.

67. Sullivan, S.; Harger, B.; Cleary, J.D. Stevens-Johnson syndrome secondary to erythromycin. Ann. Pharm. 1999, 33, 1369. [CrossRef] [PubMed]

68. Sharma, V.K.; Sethuraman, G.; Kumar, B. Cutaneous adverse drug reactions: Clinical pattern and causative agents-a 6 year series from Chandigarh, India. J. Postgrad. Med. 2001, 47, 95-99.

69. Williams, D.A. Stevens-Johnson syndrome after erythromycin therapy while deployed at sea. Mil. Med. 2000, 165, 636-637. [CrossRef]

70. San Pedro de Saenz, B.; Gomez, A.; Quiralte, J.; Florido, J.F.; Martin, E.; Hinojosa, B. FDE to macrolides. Allergy 2002, 57, 55-56.

71. Forman, R.; Koren, G.; Shear, N.H. Erythema multiforme, Stevens-Johnson syndrome and toxic epidermal necrolysis in children: A review of 10 years' experience. Drug Saf. 2002, 25, 965-972. [CrossRef]

72. Rallis, E.; Balatsouras, D.G.; Kouskoukis, C.; Verros, C.; Homsioglou, E. Drug eruptions in children with ENT infections. Int. J. Pediatric Otorhinolaryngol. 2006, 70, 53-57. [CrossRef] [PubMed]

73. Rebelo Gomes, E.; Fonseca, J.; Araujo, L.; Demoly, P. Drug allergy claims in children: From self-reporting to confirmed diagnosis. Clin. Exp. Allergy 2008, 38, 191-198. [CrossRef] [PubMed]

74. Holmes, N.E.; Hodgkinson, M.; Dendle, C.; Korman, T.M. Report of oral clarithromycin desensitization. Br. J. Clin. Pharmacol. 2008, 66, 323-324. [CrossRef] [PubMed]

75. Seitz, C.S.; Brocker, E.B.; Trautmann, A. Suspicion of macrolide allergy after treatment of infectious diseases including Helicobacter pylori: Results of allergological testing. Allergol. Immunopathol. 2011, 39, 193-199. [CrossRef] [PubMed]

76. Jakkidi, M.; Basmadjian, C.; Roy, S. An illusion of septic shock: Acute generalised exanthematous pustulosis with multiorgan dysfunction. BMJ Case Rep. 2017, 2017, 1-4. [CrossRef] [PubMed]

77. Van der Linden, P.D.; van der Lei, J.; Vlug, A.E.; Stricker, B.H. Skin reactions to antibacterial agents in general practice. J. Clin. Epidemiol. 1998, 51, 703-708. [CrossRef]

78. Price, T.A.; Tuazon, C.U. Clarithromycin-induced thrombocytopenia. Clin. Infect. Dis. 1992, 15, $563-564$. [CrossRef]

79. De Vega, T.; Blanco, S.; Lopez, C.; Pascual, E.; Sanchez, M.; Zamarron, A. Clarithromycin-induced leukocytoclastic vasculitis. Eur J. Clin. Microbiol. Infect. Dis. 1993, 12, 563. [CrossRef]

80. Oteo, J.A.; Gomez-Cadinanos, R.A.; Rosel, L.; Casas, J.M. Clarithromycin-induced thrombocytopenic purpura. Clin. Infect. Dis. 1994, 19, 1170-1171. [CrossRef]

81. Vangala, R.; Cernek, P.K. Hypersensitivity reaction to clarithromycin. Ann. Pharm. 1996, 30, 300. [CrossRef]

82. Igea, J.M.; Lazaro, M. Hypersensitivity reaction to clarithromycin. Allergy 1998, 53, 107-109. [CrossRef] [PubMed]

83. Rosina, P.; Chieregato, C.; Schena, D. Fixed drug eruption from clarithromycin. Contact Dermat. 1998, $38,105$. [CrossRef] [PubMed]

84. Gavura, S.R.; Nusinowitz, S. Leukocytoclastic vasculitis associated with clarithromycin. Ann. Pharm. 1998, 32, 543-545. [CrossRef] [PubMed] 
85. Goldberg, E.I.; Shoji, T.; Sapadin, A.N. Henoch-Schonlein purpura induced by clarithromycin. Int. J. Derm. 1999, 38, 706-708. [CrossRef] [PubMed]

86. Hamamoto, Y.; Ohmura, A.; Kinoshita, E.; Muto, M. Fixed drug eruption due to clarithromycin. Clin. Exp. Derm. 2001, 26, 48-49. [CrossRef] [PubMed]

87. Gangemi, S.; Ricciardi, L.; Fedele, R.; Isola, S.; Purello-D'Ambrosio, F. Immediate reaction to clarithromycin. Allergol. Immunopathol. 2001, 29, 31-32. [CrossRef]

88. Masia, M.; Gutierrez, F.; Jimeno, A.; Navarro, A.; Borras, J.; Matarredona, J.; Martin-Hidalgo, A. Fulminant hepatitis and fatal toxic epidermal necrolysis (Lyell disease) coincident with clarithromycin administration in an alcoholic patient receiving disulfiram. therapy. Arch. Int. Med. 2002, 162, 474-476. [CrossRef] [PubMed]

89. Borras-Blasco, J.; Enriquez, R.; Amoros, F.; Cabezuelo, J.B.; Navarro-Ruiz, A.; Perez, M.; Fernandez, J. Henoch-Schonlein purpura associated with clarithromycin. Case report and review of literature. Int. J. Clin. Pharmacol. Ther. 2003, 41, 213-216. [CrossRef]

90. Terzano, C.; Petroianni, A. Clarithromycin and pulmonary infiltration with eosinophilia. BMJ 2003, 326, 1377-1378. [CrossRef]

91. Baz, K.; Ikizoglu, G.; Yazici, A.C.; Kokturk, A.; Tiftik, N.; Apa, D.D.; Demirseren, D. Fatal aplastic anaemia in a patient with clarithromycin-induced toxic epidermal necrolysis. J. Eur. Acad. Derm. Venereol. 2004, 18, $104-105$. [CrossRef]

92. Ohnishi, H.; Abe, M.; Yokoyama, A.; Hamada, H.; Ito, R.; Hirayama, T.; Nishimura, K.; Higaki, J. Clarithromycin-induced eosinophilic pneumonia. Int. Med. 2004, 43, 231-235. [CrossRef] [PubMed]

93. Alonso, J.C.; Melgosa, A.C.; Gonzalo, M.J.; Garcia, C.M. Fixed drug eruption on the tongue due to clarithromycin. Contact Dermat. 2005, 53, 121-122. [CrossRef] [PubMed]

94. Khaldi, N.; Miras, A.; Gromb, S. Toxic epidermal necrolysis and clarithromycin. Can J. Clin. Pharmcol. 2005, 12, e264-e268.

95. Dore, J.; Salisbury, R.E. Morbidity and mortality of mucocutaneous diseases in the pediatric population at a tertiary care center. J. Burn Care Res. 2007, 28, 865-870. [CrossRef] [PubMed]

96. Clayton, T.H.; Barry, J.; Fitzgerald, D.; Watson, R.; Irvine, A.D. Clarithromycin suspension-associated toxic epidermal necrolysis in a 2-year-old girl. Clin. Exp. Derm. 2007, 32, 755-756. [CrossRef] [PubMed]

97. Lange, L.; Koningsbruggen, S.V.; Rietschel, E. Questionnaire-based survey of lifetime-prevalence and character of allergic drug reactions in German children. Pediatr Allergy Immunol. 2008, 19, 634-638. [CrossRef] [PubMed]

98. Ben-Shoshan, M.; Moore, A.; Primeau, M.N. Anaphylactic reaction to clarithromycin in a child. Allergy 2009, 64, 962-963. [CrossRef]

99. Mori, F.; Barni, S.; Pucci, N.; Rossi, E.; Azzari, C.; de Martino, M.; Novembre, E. Sensitivity and specificity of skin tests in the diagnosis of clarithromycin allergy. Ann. Allergy Asthma Immunol. 2010, 104, 417-419. [CrossRef]

100. Swamy, N.; Laurie, S.A.; Ruiz-Huidobro, E.; Khan, D.A. Successful clarithromycin desensitization in a multiple macrolide-allergic patient. Ann. Allergy Asthma Immunol. 2010, 105, 489-490. [CrossRef]

101. Mittmann, N.; Knowles, S.R.; Koo, M.; Shear, N.H.; Rachlis, A.; Rourke, S.B. Incidence of toxic epidermal necrolysis and Stevens-Johnson Syndrome in an HIV cohort: An observational, retrospective case series study. Am. J. Clin. Derm. 2012, 13, 49-54. [CrossRef]

102. Petitto, J.; Chervinskiy, S.K.; Scurlock, A.M.; Perry, T.T.; Jones, S.M.; Pesek, R.D. Successful clarithromycin desensitization in a macrolide-sensitive pediatric patient. J. Allergy Clin. Immunol. Pract. 2013, 1, 307-308. [CrossRef] [PubMed]

103. Malkarnekar, S.B.; Naveen, L. Fixed drug eruption due to clarithromycin. J. Res. Pharm. Pract. 2013, 2, 169-171. [CrossRef] [PubMed]

104. Barni, S.; Butti, D.; Mori, F.; Pucci, N.; Rossi, M.E.; Cianferoni, A.; Novembre, E. Azithromycin is more allergenic than clarithromycin in children with suspected hypersensitivity reaction to macrolides. J. Investig. Allergol. Clin. Immunol. 2015, 25, 128-132. [PubMed]

105. Blair, P.W.; Herrin, D.; Abaalkhail, N.; Fiser, W. DRESS syndrome presenting after initiation of mycobacterium avium complex osteomyelitis treatment. BMJ Case Rep. 2015, 2015.

106. Guvenir, H.; Dibek Misirlioglu, E.; Capanoglu, M.; Vezir, E.; Toyran, M.; Kocabas, C.N. Proven Non-beta-LactAm. Antibiotic Allergy in Children. Int. Arch. Allergy Immunol. 2016, 169, 45-50. [CrossRef] [PubMed] 
107. Trevisi, P.; Patrizi, A.; Neri, I.; Farina, P. Toxic pustuloderma associated with azithromycin. Clin. Exp. Derm. 1994, 19, 280-281. [CrossRef] [PubMed]

108. Naldi, L.; Conforti, A.; Venegoni, M.; Troncon, M.G.; Caputi, A.; Ghiotto, E.; Cocci, A.; Moretti, U.; Velo, G.; Leone, R. Cutaneous reactions to drugs. An analysis of spontaneous reports in four Italian regions. Br. J. Clin. Pharmacol. 1999, 48, 839-846. [CrossRef] [PubMed]

109. Schissel, D.J.; Singer, D.; David-Bajar, K. Azithromycin eruption in infectious mononucleosis: A proposed mechanism of Interaction. Cutis 2000, 65, 163-166. [PubMed]

110. Cascaval, R.I.; Lancaster, D.J. Hypersensitivity syndrome associated with azithromycin. Am. J. Med. 2001, 110, 330-331. [CrossRef]

111. Dakdouki, G.K.; Obeid, K.H.; Kanj, S.S. Azithromycin-induced rash in infectious mononucleosis. Scand J. Infect. Dis. 2002, 34, 939-941. [CrossRef]

112. Taylor, W.R.; Richie, T.L.; Fryauff, D.J.; Ohrt, C.; Picarima, H.; Tang, D.; Murphy, G.S.; Widjaja, H.; Braitman, D.; Tjitra, E.; et al. Tolerability of azithromycin as malaria prophylaxis in adults in northeast papua, indonesia. Antimicrob. Agents Chemother. 2003, 47, 2199-2203. [CrossRef] [PubMed]

113. Odemis, E.; Kalyoncu, M.; Okten, A.; Yildiz, K. Azithromycin-induced leukocytoclastic vasculitis. J. Rheumatol. 2003, 30, 2292. [PubMed]

114. Aihara, Y.; Ito, S.; Kobayashi, Y.; Aihara, M. Stevens-Johnson syndrome associated with azithromycin followed by transient reactivation of herpes simplex virus infection. Allergy 2004, 59, 118. [CrossRef] [PubMed]

115. Noel, M.V.; Sushma, M.; Guido, S. Cutaneous adverse drug reactions in hospitalized patients in a tertiary care center. Indian J. Pharmacol. 2004, 36, 292-295.

116. Brkljacic, N.; Gracin, S.; Prkacin, I.; Sabljar-Matovinovic, M.; MrzlJak, A.; Nemet, Z. Stevens-Johnson syndrome as an unusual adverse effect of azithromycin. Acta Derm. Croat. 2006, 14, 40-45.

117. Cummings, J.E.; Snyder, R.R.; Kelly, E.B.; Raimer, S.S. Drug-induced linear immunoglobulin A bullous dermatosis mimicking Stevens-Johnson syndrome: A case report. Cutis 2007, 79, 203-207. [PubMed]

118. Pursnani, A.; Yee, H.; Slater, W.; Sarswat, N. Hypersensitivity myocarditis associated with azithromycin exposure. Ann. Int. Med. 2009, 150, 225-226. [CrossRef]

119. Flavia Monteagudo Paz, A.; Francisco Silvestre Salvador, J.; Latorre Martinez, N.; Cuesta Montero, L.; Toledo Alberola, F. Allergic contact dermatitis caused by azithromycin in an eye drop. Contact Dermat. 2011, 64, 300-301. [CrossRef] [PubMed]

120. Bauer, K.A.; Brimhall, A.K.; Chang, T.T. Drug reaction with eosinophilia and Systemic symptoms (DRESS) associated with azithromycin in acute Epstein-Barr virus infection. Pediatric Derm. 2011, 28, 741-743. [CrossRef]

121. Mori, F.; Pecorari, L.; Pantano, S.; Rossi, M.E.; Pucci, N.; De Martino, M.; Novembre, E. Azithromycin anaphylaxis in children. Int. J. Immunopathol. Pharmacol. 2014, 27, 121-126. [CrossRef]

122. Banerjee, I.; Mondal, S.; Sen, S.; Tripathi, S.K.; Banerjee, G. Azithromycin-induced rash in a patient of infectious mononucleosis-A case report with review of literature. J. Clin. Diagn. Res. 2014, 8, HD01-HD02. [CrossRef] [PubMed]

123. Mendes-Bastos, P.; Bras, S.; Amaro, C.; Cardoso, J. Non-occupational allergic contact dermatitis caused by azithromycin in an eye solution. J. Dtsch. Derm. Ges. 2014, 12, 729-730. [CrossRef] [PubMed]

124. Sriratanaviriyakul, N.; Nguyen, L.P.; Henderson, M.C.; Albertson, T.E. Drug reaction with eosinophilia and Systemic symptoms syndrome (DRESS) syndrome associated with azithromycin presenting like septic shock: A case report. J. Med. Case Rep. 2014, 8, 332. [CrossRef] [PubMed]

125. Da Cunha Filho, R.R.; Bordignon, S.C.; Cassol, M.; Rastelli, G.J. Acute generalized exanthematous pustulosis by azithromycin. Int. J. Derm. 2015, 54, e247-e249. [CrossRef] [PubMed]

126. Das, A.; Sancheti, K.; Podder, I.; Das, N.K. Azithromycin induced bullous fixed drug eruption. Indian J. Pharmacol. 2016, 48, 83-85. [CrossRef] [PubMed]

127. Nappe, T.M.; Goren-Garcia, S.L.; Jacoby, J.L. Stevens-Johnson syndrome after treatment with azithromycin: An uncommon culprit. Am. J. Emerg. Med. 2016, 34, 676 e1-3. [CrossRef] [PubMed]

128. Campanon-Toro, M.V.; Sierra, O.; Moreno, E.; Sobrino-Garcia, M.; Gracia-Bara, M.T.; Davila, I. Acute generalized exanthematous pustulosis (AGEP) induced by azithromycin. Contact Dermat. 2017, 76, 363-364. [CrossRef]

129. Kobayashi, A.; Takasawa, R.; Takasawa, K.; Nishioka, M.; Kaneko, M.; Ono, H.; Maekawa, T.; Morio, T.; Shimohira, M. An infant case of severe hypereosinophilia and Systemic symptoms with multiple drug hypersensitivity and reactivation of cytomegalovirus and BK virus. Allergol. Int. 2017, 66, 479-481. [CrossRef] 
130. An, I.; Demir, V.; Akdeniz, S. Fixed drug eruption probably induced by azithromycin. Australas J. Derm. 2017, 58, e253-e254. [CrossRef]

131. Xu, L.; Zhu, Y.; Yu, J.; Deng, M.; Zhu, X. Nursing care of a boy seriously infected with Steven-Johnson syndrome after treatment with azithromycin: A case report and literature review. Medicine (Baltimore) 2018, 97, e9112. [CrossRef]

132. Iarikov, D.E.; Alexander, J.; Nambiar, S. Hypersensitivity reactions associated with fidaxomicin use. Clin. Infect. Dis. 2014, 58, 537-539. [CrossRef] [PubMed]

133. Park, J.; Matsui, D.; Rieder, M.J. Multiple antibiotic sensitivity syndrome in children. Can. J. Clin. Pharmacol. 2000, 7, 38-41. [PubMed]

134. Messaad, D.; Sahla, H.; Benahmed, S.; Godard, P.; Bousquet, J.; Demoly, P. Drug provocation tests in patients with a history suggesting an immediate drug hypersensitivity reaction. Ann. Int. Med. 2004, 140, 1001-1006. [CrossRef] [PubMed]

(C) 2019 by the authors. Licensee MDPI, Basel, Switzerland. This article is an open access article distributed under the terms and conditions of the Creative Commons Attribution (CC BY) license (http://creativecommons.org/licenses/by/4.0/). 